\title{
Les conditions météorologiques propices au déclenchement des avalanches de neige dans les corridors routiers du nord de la Gaspésie, Québec, Canada Analysis of the weather conditions leading to avalanche activity along transportation roads in Northern Gaspésie, Québec, Canada
}

\section{Bernard Hétu}

Volume 61, numéro 2-3, 2007

URI : https://id.erudit.org/iderudit/038990ar

DOI : https://doi.org/10.7202/038990ar

Aller au sommaire du numéro

Éditeur(s)

Les Presses de l'Université de Montréal

ISSN

0705-7199 (imprimé)

1492-143X (numérique)

Découvrir la revue

Citer cet article

Hétu, B. (2007). Les conditions météorologiques propices au déclenchement des avalanches de neige dans les corridors routiers du nord de la Gaspésie, Québec, Canada. Géographie physique et Quaternaire, 61(2-3), 165-180.

https://doi.org/10.7202/038990ar

\section{Résumé de l'article}

Le nord de la Gaspésie constitue l'une des principales régions à risque d'avalanche au Québec. Le risque ne concerne pas uniquement les adeptes de la montagne, mais également les véhicules qui circulent sur les routes nationales 132 et 198 entre Sainte-Anne-des-Monts et Grande-Vallée. Chaque hiver, des masses de neige recouvrent partiellement ou complètement la chaussée, interrompant la circulation et causant parfois des accidents. Cet article a pour objectifs de faire connaître les principales zones à risque d'avalanche dans les corridors routiers du nord de la Gaspésie et d'identifier les conditions météorologiques propices au déclenchement des avalanches dans le contexte climatique du nord de la Gaspésie. Trois séries de données ont été analysées, soit les résultats d'un inventaire de l'activité avalancheuse effectué par le Ministère des Transports du Québec entre 1987 et 1993, une série d'événements avalancheux ponctuels ayant causé des accidents routiers, dont trois mortels (1935, 1956 et 1971), pour un total de 21 journées avalancheuses. Les dates d'avalanche ont été confrontées aux données météorologiques fournies par les stations d'Environnement Canada. Il semble, à la lumière des données disponibles, que les versants côtiers du nord de la Gaspésie connaissent deux principaux régimes avalancheux : un régime printanier associé à la fonte, à la pluie et au démantèlement des carapaces de glace qui se forment sur les parois rocheuses durant l'hiver et un régime hivernal modulé par les tempêtes de neige. 


\section{LES CONDITIONS MÉTÉOROLOGIQUES PROPICES AU DÉCLENCHEMENT DES AVALANCHES DE NEIGE DANS LES CORRIDORS ROUTIERS DU NORD DE LA GASPÉSIE, QUÉBEC, CANADA}

Bernard HÉTU*, Groupe de recherche Bionord et Centre d'études nordiques, Département de biologie, chimie et géographie, Université du Québec à Rimouski, 300 allée des Ursulines, C.P. 3300, Rimouski, Québec G5L 3A1, Canada.

RÉSUMÉ. Le nord de la Gaspésie constitue l'une des principales régions à risque d'avalanche au Québec. Le risque ne concerne pas uniquement les adeptes de la montagne, mais également les véhicules qui circulent sur les routes nationales 132 et 198 entre SainteAnne-des-Monts et Grande-Vallée. Chaque hiver, des masses de neige recouvrent partiellement ou complètement la chaussée, interrompant la circulation et causant parfois des accidents. Cet article a pour objectifs de faire connaître les principales zones à risque d'avalanche dans les corridors routiers du nord de la Gaspésie et d'identifier les conditions météorologiques propices au déclenchement des avalanches dans le contexte climatique du nord de la Gaspésie. Trois séries de données ont été analysées, soit les résultats d'un inventaire de l'activité avalancheuse effectué par le Ministère des Transports du Québec entre 1987 et 1993, une série d'événements avalancheux ponctuels ayant causé des accidents routiers, dont trois mortels (1935, 1956 et 1971), pour un total de 21 journées avalancheuses. Les dates d'avalanche ont été confrontées aux données météorologiques fournies par les stations d'Environnement Canada. II semble, à la lumière des données disponibles, que les versants côtiers du nord de la Gaspésie connaissent deux principaux régimes avalancheux : un régime printanier associé à la fonte, à la pluie et au démantèlement des carapaces de glace qui se forment sur les parois rocheuses durant l'hiver et un régime hivernal modulé par les tempêtes de neige.
ABSTRACT. Analysis of the weather conditions leading to avalanche activity along transportation roads in Northern Gaspésie, Québec, Canada. Northern Gaspésie is one the principal areas of snow avalanche risk in Québec. Snow avalanche hazard represents a treat to back-country recreational activities, but also to the vehicles circulating on national roads 132 and 198 between Sainte-Anne-des-Monts and Grande-Vallée. Each winter, masses of snow cover partially or completely the roadway, stopping circulation and causing accidents sometimes. This paper aims to localize the main avalanche risk zones into Northern Gaspésie transportation corridors and the analysis of the weather situations leading to avalanche activity along transportation roads under the specific climate of Northern Gaspésie. Three sets of data were gathered together, an inventory of avalanche activity realized by the Ministère des Transports du Québec between 1987 and 1993, a series of specific avalanche events having caused road accidents, including three mortals (1935, 1956 and 1971), for a total of 21 avalanche days, and the dates of avalanches were confronted with weather data provided by the stations of Environment Canada. Preliminary results indicate that the coastal slopes of Northern Gaspésie have two major avalanche regimes: a spring regime associated with snowmelt, rain and ice-block fall from rock walls, and a winter regime associated with snowstorms. 


\section{INTRODUCTION}

Compte tenu de son relief accidenté et des fortes précipitations neigeuses qui la caractérisent, la Gaspésie constitue l'une des principales régions à risque d'avalanche au Québec (Atlas du Bas-Saint-Laurent, 1998; Hétu et Bergeron, 2004; Hétu et Brown, 2006). Le risque concerne non seulement les skieurs hors-piste qui fréquentent les Chic-Chocs (Boucher, 2000 ; Boucher et Gagnon, 2002 ; Boucher et al. 2003 ; Gagnon 2003), mais également plusieurs segments de routes localisés au pied de pentes avalancheuses notoires (Girard et Hétu, 1994; Hétu et al., 1994). Les routes nationales 132 et 198, entre Sainte-Anne-des-Monts et Grande-Vallée, y sont particulièrement exposées (Transports Québec, 2005). Régulièrement, des masses de neige recouvrent partiellement ou complètement la chaussée, interrompant la circulation et causant parfois des accidents. Bien que la probabilité qu'un véhicule soit heurté directement par une avalanche en mouvement soit relativement faible (McClung, 1999), le risque est bien réel comme l'attestent les accidents routiers décrits plus loin. De plus, l'expérience démontre que le risque d'accident perdure même après l'avalanche: les masses de neige compactes qui obstruent la chaussée représentent en effet de véritables pièges pour les véhicules qui viennent s'y emboutir, particulièrement lorsque les avalanches surviennent la nuit ou quand la visibilité est réduite ou nulle (tempête, blizzard, brouillard côtier), comme ce fut le cas le 20 janvier 2004 près de Mont-Saint-Pierre (Alain Dumont, verbatim). II est donc nécessaire d'accroître les connaissances sur les avalanches qui menacent les routes gaspésiennes. Cet article a deux objectifs: (1) faire connaître les principales zones à risque dans les corridors routiers du nord de la Gaspésie et (2) identifier les conditions météorologiques propices au déclenchement des avalanches dans le contexte climatique spécifique de la zone littorale de la Haute-Gaspésie.

Dans le cadre de cette étude, le mot «hiver» désigne la saison avalancheuse qui peut durer jusqu'à sept mois certaines années, soit d'octobre à mai inclusivement. Par exemple, l'hiver 1955-1956 commence en octobre 1955 et se termine en mai 1956. À moins d'avis contraire, la quantité de neige tombée durant l'hiver correspond au cumul des précipitations observées durant la saison avalancheuse (octobre à mai).

\section{TRAVAUX ANTÉRIEURS}

La Gaspésie compte quelques centaines de couloirs d'avalanche qui se concentrent dans quatre zones distinctes (Veillette et Cloutier, 1993): les monts Chic-Chocs (incluant le mont Albert), les monts McGerrigle, la vallée de la Cascapédia ainsi que l'escarpement littoral et les basses vallées de la région de Mont-Louis (fig. 1).

L'existence des avalanches en Gaspésie est mentionnée dès le début des années 1960 dans une brève note publiée par Gaumond et Hamelin (1960), mais il faudra attendre les années 1980 avant que ne soient réalisées les premières

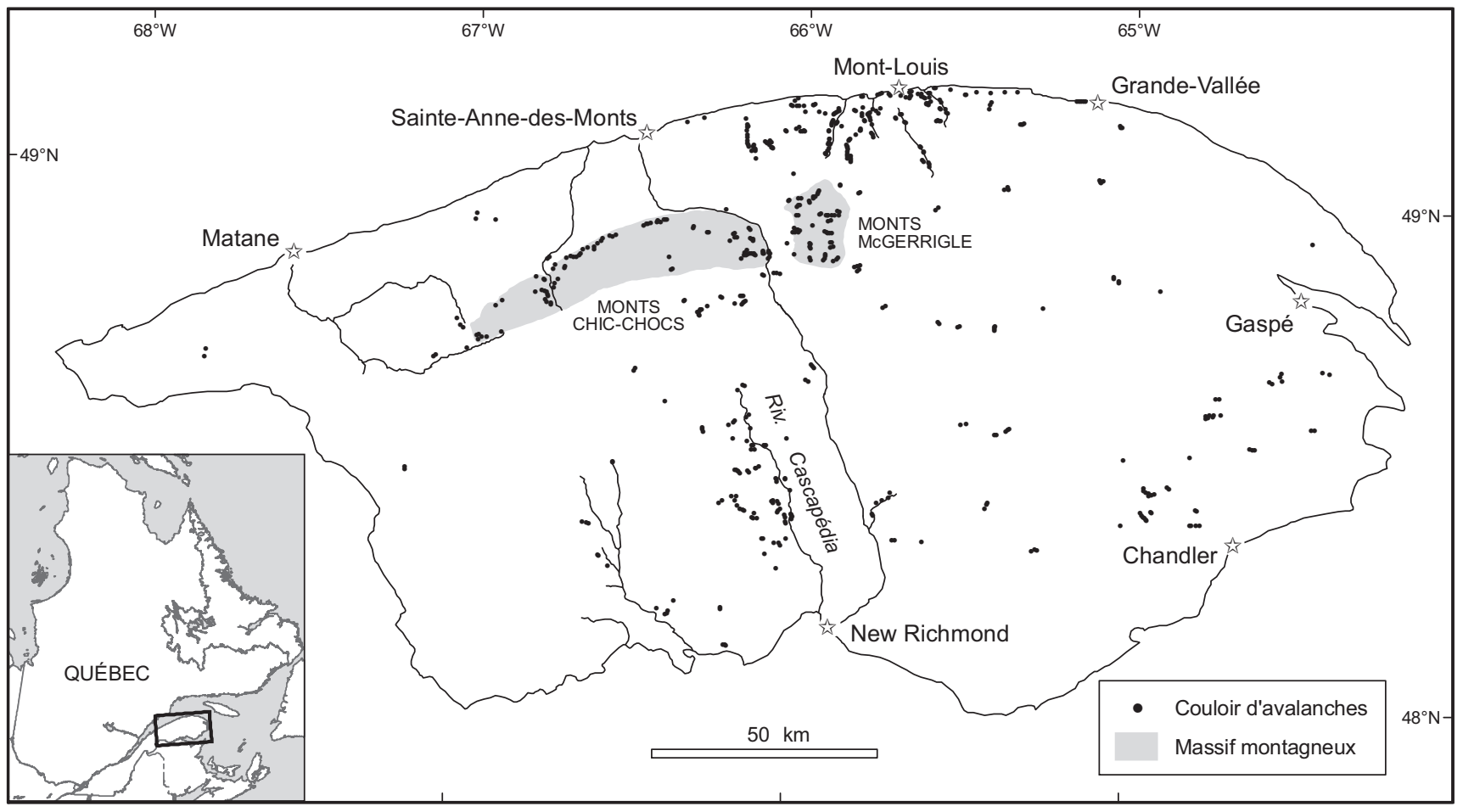

FIGURE 1. Les couloirs d'avalanche de la Gaspésie se concentrent dans quatre secteurs principaux: la région de Mont-Louis (cette étude), les monts Chic-Chocs, les monts McGerrigle et la vallée de la rivière Cascapédia.
Avalanche paths in Gaspésie are concentrated in four major areas: the Mont-Louis region (this study), the Chic-Chocs Mountains, the McGerrigle Mountains and the Cascapedia River Valley. 
études portant spécifiquement sur ce phénomène. Plusieurs études visant à préciser la répartition, la fréquence et les impacts écologiques et géomorphologiques des avalanches ont été effectuées à la fois dans les massifs montagneux de la Gaspésie centrale (Girard et Hétu, 1989; Hétu et al., 1994; Larocque et al., 2001; Talbot, 2002 ; Boucher et al., 2003; Germain, 2005; Royer et Lemieux, 2006; Lemieux, 2007) et dans la zone côtière entre Sainte-Anne-des-Monts et GrandeVallée (Hétu, 1986, 1990, 1991, 1995; Hétu et Vandelac, 1989; Dubé et al., 2004 ; Germain, 2005; Germain et al., 2005).

Les études dendrochronologiques effectuées par Dubé et al. (2004) sur quelques talus d'éboulis des vallées de MontSaint-Pierre (2 talus) et de Rivière-à-Claude (1 talus) ont permis de reconstituer la fréquence des grosses avalanches de neige entre 1860 et 1997. D'un versant à l'autre, le nombre d'années avalancheuses identifiées varie du simple au double (entre 6 et 11), mais à l'échelle régionale, sept hivers se distinguent par l'étendue et l'ampleur des dommages causés à la végétation ligneuse par les avalanches: 1870-1871, 18971898, 1951-1952, 1955-1956, 1965-1966, 1976-1977 et 19951996. Les données dendrochronologiques ne permettent pas de préciser si les dommages se rapportant à une année en particulier sont attribuables à une avalanche majeure ou à une série d'avalanches de taille réduite, mais nous savons en revanche que six des sept hivers de forte activité avalancheuse se distinguent par des chutes de neige largement supérieures à la moyenne (tabl. I). Par exemple, au cours des hivers 1951-1952 et 1955-1956, les chutes de neige atteignaient à Cap-Madeleine, respectivement, 695,9 cm et $551,8 \mathrm{~cm}$, alors que la moyenne pour la période de 1885 à 2000 est de 330,1 cm (Environnement Canada, 2005a).
L'étude réalisée par Dubé et al. (2004) laisse entrevoir une relation entre la quantité de neige tombée et l'activité avalancheuse, mais comme la dendrochronologie ne permet pas de connaître la date des avalanches, il est impossible de pousser l'analyse plus loin. Or tout programme de prévention des risques associés à l'activité avalancheuse implique une bonne connaissance à la fois des caractéristiques du manteau neigeux et des conditions météorologiques propices au déclenchement des avalanches (McClung et Schaerer, 2006). Bien qu'une vaste expertise ait été développée sur ces aspects dans l'ouest du continent nord-américain (Fitzharris, 1981; Mock et Birkeland, 2000; Hägeli et McClung, 2003), il est difficile de la transposer directement dans l'est canadien puisque le climat, le relief et les conditions d'enneigement y sont fort différents.

\section{SECTEUR D'ÉTUDE}

\section{ESCARPEMENT LITTORAL ET BASSES VALLÉES DU NORD DE LA GASPÉSIE}

Cette étude concerne plus particulièrement deux segments de route distincts, soit la route nationale 132 entre SainteAnne-des-Monts et Grande-Vallée et la route nationale 198 dans le secteur du lac de l'Anse-Pleureuse (fig. 2). Dans cette région du nord de la Gaspésie, le relief se caractérise par un vaste plateau boisé qui est recoupé par un escarpement côtier imposant et par des vallées profondes et étroites, bordées de versants raides, ce qui laisse peu de place au réseau routier. À plusieurs endroits, les routes, coincées entre la montagne et la mer ou un lac, sont situées directement au pied des pentes avalancheuses - comme c'est le cas le long du lac de l'Anse-Pleureuse - ou, au plus, à une dizaine de mètres des versants, parfois moins (fig. 2 et 3 ). Le long du littoral, les

\section{TABLEAU I}

Précipitations totales, nombre et intensité des tempêtes de neige durant les hivers de forte activité avalancheuse selon les analyses dendrochronologiques de Dubé et al. (2004) et les années couvertes par l'inventaire de la Patrouille de Roches du Ministère des Transports du Québec (source des données météorologiques: Environnement Canada, 2005a).

\begin{tabular}{|c|c|c|c|c|c|}
\hline \multirow{2}{*}{ Hiver } & \multicolumn{2}{|c|}{ Nombre de tempêtes } & \multirow{2}{*}{$\begin{array}{l}\text { Nombre total } \\
\text { de tempêtes }\end{array}$} & \multirow{2}{*}{$\begin{array}{c}\text { Précipitations } \\
\text { neigeuses totales } \\
\text { durant l'hiver }(\mathrm{cm})^{\star}\end{array}$} & \multirow{2}{*}{$\begin{array}{c}\text { Nombre } \\
\text { d'avalanches }\end{array}$} \\
\hline & $>10 \mathrm{~cm} / \mathrm{jour}$ & $>25 \mathrm{~cm} / \mathrm{jour}$ & & & \\
\hline \multicolumn{6}{|c|}{ Dubé et al. (2004) } \\
\hline $1870-1871$ & - & - & - & - & - \\
\hline 1897-1898 & 6 & 5 & 11 & 289,1 & - \\
\hline 1951-1952 & 20 & 3 & 23 & 695,9 & - \\
\hline 1955-1956 & 14 & 6 & 20 & 551,8 & - \\
\hline 1965-1966 & 12 & 2 & 14 & 412,5 & - \\
\hline 1976-1977 & 2 & 0 & 2 & 363,9 & - \\
\hline 1995-1996 & 10 & 3 & 13 & $432,6^{\star \star}$ & - \\
\hline \multicolumn{6}{|c|}{ Ministère des transports du Québec } \\
\hline $1987-1988$ & 12 & 1 & 13 & 314,8 & 8 \\
\hline 1988-1989 & 8 & 0 & 8 & 253,8 & 4 \\
\hline 1989-1990 & 6 & 1 & 7 & 215,4 & 0 \\
\hline 1990-1991 & 8 & 4 & 12 & 373,8 & 3 \\
\hline 1991-1992 & 10 & 0 & 10 & 267,0 & 2 \\
\hline $1992-1993$ & 9 & 0 & 9 & 271,6 & 2 \\
\hline
\end{tabular}

\footnotetext{
* Total des mois d'octobre à mai inclusivement.

${ }^{* *}$ Cette valeur provient de la station de Grande-Vallée.
} 

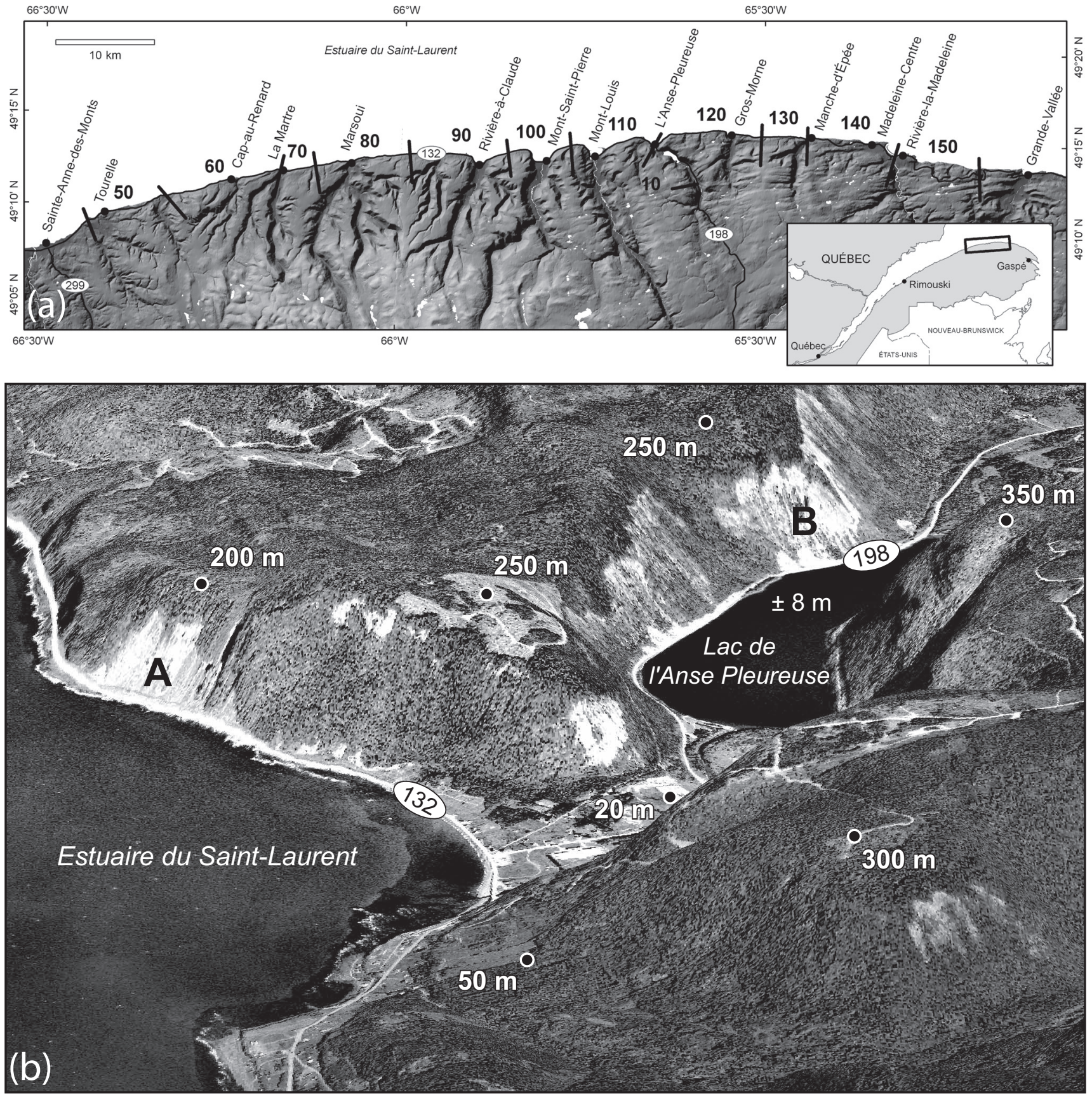

FIGURE 2. (A) Les 12 secteurs d'inventaire des avalanches du Ministère des Transports du Québec. (B) Dans le nord de la Gaspésie, les routes nationales 132 et 198 sont localisées directement au pied des pentes avalancheuses. Les lettres A (secteur 120) et B (secteur 10) localisent les principales zones à risque dans la vallée de l'AnsePleureuse.

pentes avalancheuses étudiées sont orientées vers le nord $\left(0^{\circ} \pm 45^{\circ}\right)$, alors que dans la vallée de l'Anse-Pleureuse, elles sont orientées vers le sud-ouest.

Les pentes avalancheuses de la région peuvent être regroupées en deux catégories: les talus d'éboulis et les étroits couloirs à carapace de glace.
(A) The twelve avalanche inventory zones of the Ministère des Transports du Québec. (B) In Northern Gaspésie, national roads 132 and 198 are located directly at the foot of the avalanche-prone slopes. Letters $A$ (section 120) and B (section 10) locate the main avalancheprone areas in the Anse-Pleureuse Valley.

Talus d'éboulis actifs

La plupart des pentes avalancheuses correspondent en fait à des talus d'éboulis dont la dénivelée, mesurée entre le pied de la paroi et la base du versant, varie entre $50 \mathrm{~m}$ et un peu plus de 300 m (Hétu et Gray, 1980; Hétu et Vandelac, 1989; Hétu, 1991). L'inclinaison des talus d'éboulis passe de 37 à $42^{\circ}$ près 

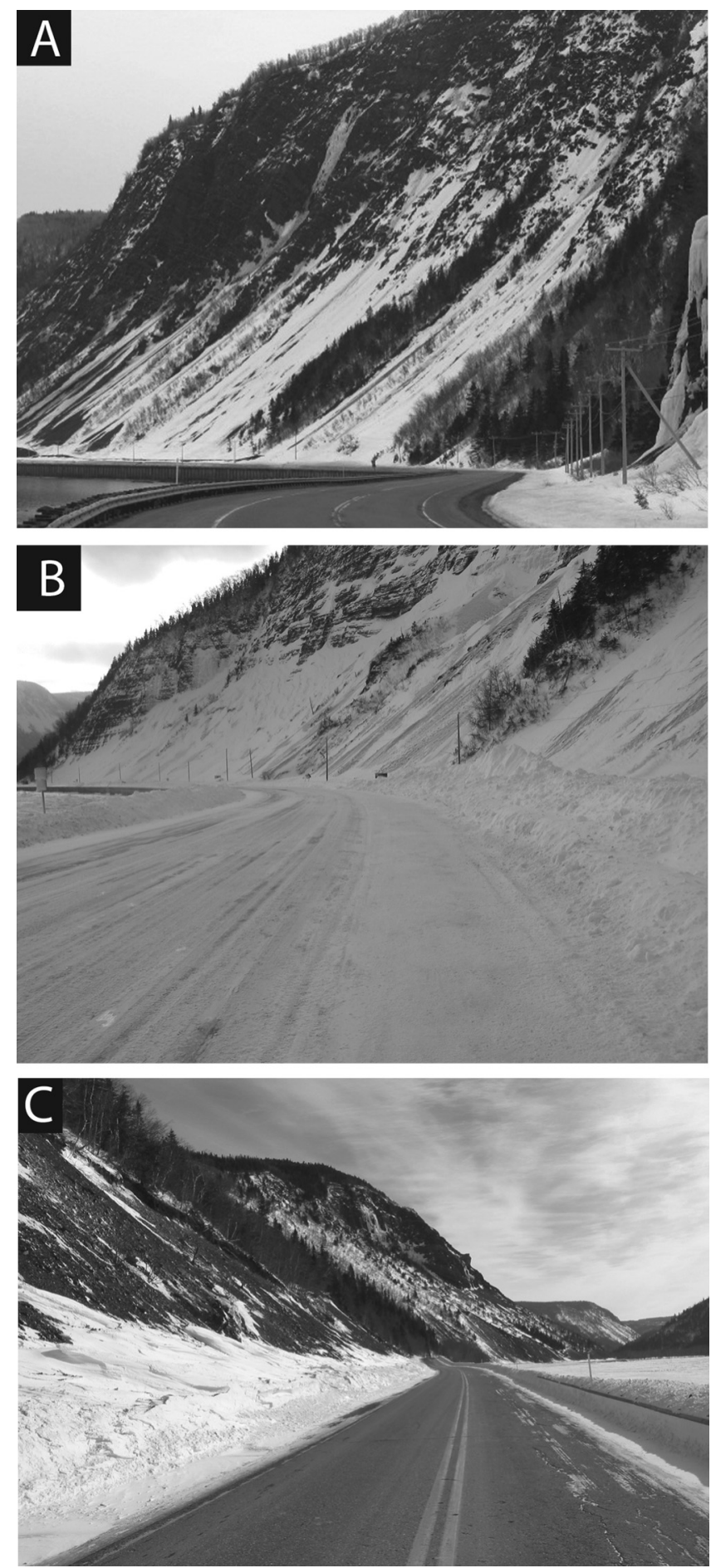

FIGURE 3. Dans le nord de la Gaspésie, la plupart des avalanches démarrent sur les talus d'éboulis qui dominent les routes nationales 132 et 198. Les pentes d'avalanches le long (A) de la route nationale 132 à l'est de Gros-Morne (secteur 130), (B) de la route nationale 132 à l'ouest de Mont-Saint-Pierre (secteur 100) et (C) de la route nationale 198 le long du lac de l'Anse-Pleureuse (secteur 10).

In Northern Gaspésie, the majority of the avalanches start on the scree-slopes. Avalanche slopes along (A) the national road 132 near Gros-Morne (section 130), (B) the national road 132 near Mont-SaintPierre (section 100) and (C) the national road 198 near AnsePleureuse (section 10). de la paroi à moins de $30^{\circ}$ dans la partie inférieure. Leur extension latérale est très variable puisqu'elle va de quelques dizaines de mètres à quelques centaines de mètres (fig. 2 et 3). Les talus d'éboulis sont particulièrement fréquents sur la côte entre l'Anse-Pleureuse et Manche-d'Épée ainsi que de part et d'autre de la baie de Mont-Saint-Pierre. Ils sont très nombreux également dans certaines vallées, notamment à l'AnsePleureuse où ils sont particulièrement étendus (fig. 2B et $3 \mathrm{C}$ ). Sur la côte, la plupart des talus d'éboulis actifs sont totalement dénués de végétation, depuis la paroi jusqu'au pied du versant, tandis que ceux qui dominent la route nationale 198 à l'AnsePleureuse sont largement boisés, mais il subsiste des couloirs arbustifs et des zones d'éboulis vif qui permettent le départ des avalanches (fig. 2B et 3C). Les éboulis avalancheux de l'AnsePleureuse sont recoupés à la base par un talus artificiel fortement incliné (emprise de la route) qui leur confère un profil convexe, ce qui en accroît l'instabilité (fig. 3C).

\section{Couloirs à carapace de glace}

À l'ouest de Rivière-à-Claude, les talus d'éboulis actifs sont plutôt rares. Les avalanches se produisent dans d'étroits couloirs encadrés par la forêt et dont la largeur est généralement inférieure à 20 m (fig. 4). Plusieurs couloirs d'avalanche débouchent directement sur la route nationale 132. Longs de $50 \mathrm{~m}$ à plus $250 \mathrm{~m}$, leur pente moyenne varie entre moins de $30^{\circ}$ et un peu plus de $37^{\circ}$ (en excluant la paroi rocheuse, beaucoup plus raide). La plupart des couloirs sont dominés à l'amont par une paroi rocheuse quasi-verticale de 10 à $50 \mathrm{~m}$ de dénivelée (fig. 4). Ces couloirs polygéniques connaissent une dynamique à la fois intense et originale (Groleau, 1994 ; Jacob, 2001). Outre les avalanches, deux processus majeurs, qui représentent eux aussi un risque pour la route, y ont été observés, soit la chute de blocs de glace et les coulées de débris (Hétu et al., 1994). De nombreuses parois à exposition nord se couvrent à chaque hiver d'épaisses carapaces de glace qui résultent de la congélation de l'eau hydrogéologique (Pelletier, 1997; fig. 4C). La chute et la fragmentation de ces carapaces entre la mi-avril et la mi-mai (Girard et Hétu, 1994) libèrent des blocs de glace pouvant atteindre 2 à $3 \mathrm{~m}$ de côté qui dévalent la pente en glissant et en bondissant (fig. 4D). L'été, les pluies torrentielles y déclenchent des coulées de débris; elles construisent des cônes alluviaux qui peuvent parfois bloquer la route.

\section{CLIMAT DE LA ZONE LITTORALE NORD-GASPÉSIENNE}

Situé en position de moyenne latitude $\left(49^{\circ} \mathrm{N}\right)$, sous la double influence des masses d'air arctiques et des dépressions en provenance de la côte atlantique des États-Unis, et, par surcroît, dans un contexte qui juxtapose la montagne et l'estuaire du Saint-Laurent, le nord de la Gaspésie possède un climat très contrasté où se conjuguent les influences maritimes, continentales et orographiques (Gagnon, 1970). Le climat de la zone côtière nord-gaspésienne est de type tempéré humide à hiver froid. Le tableau II résume les principales caractéristiques du régime climatique annuel à la station de Cap-Madeleine, située à $20 \mathrm{~m}$ d'altitude à l'extrémité est du territoire à l'étude (secteur 140: $49^{\circ} 13^{\prime} \mathrm{N}, 65^{\circ} 19^{\prime} \mathrm{O}$ ). Trois points retiennent l'attention: 

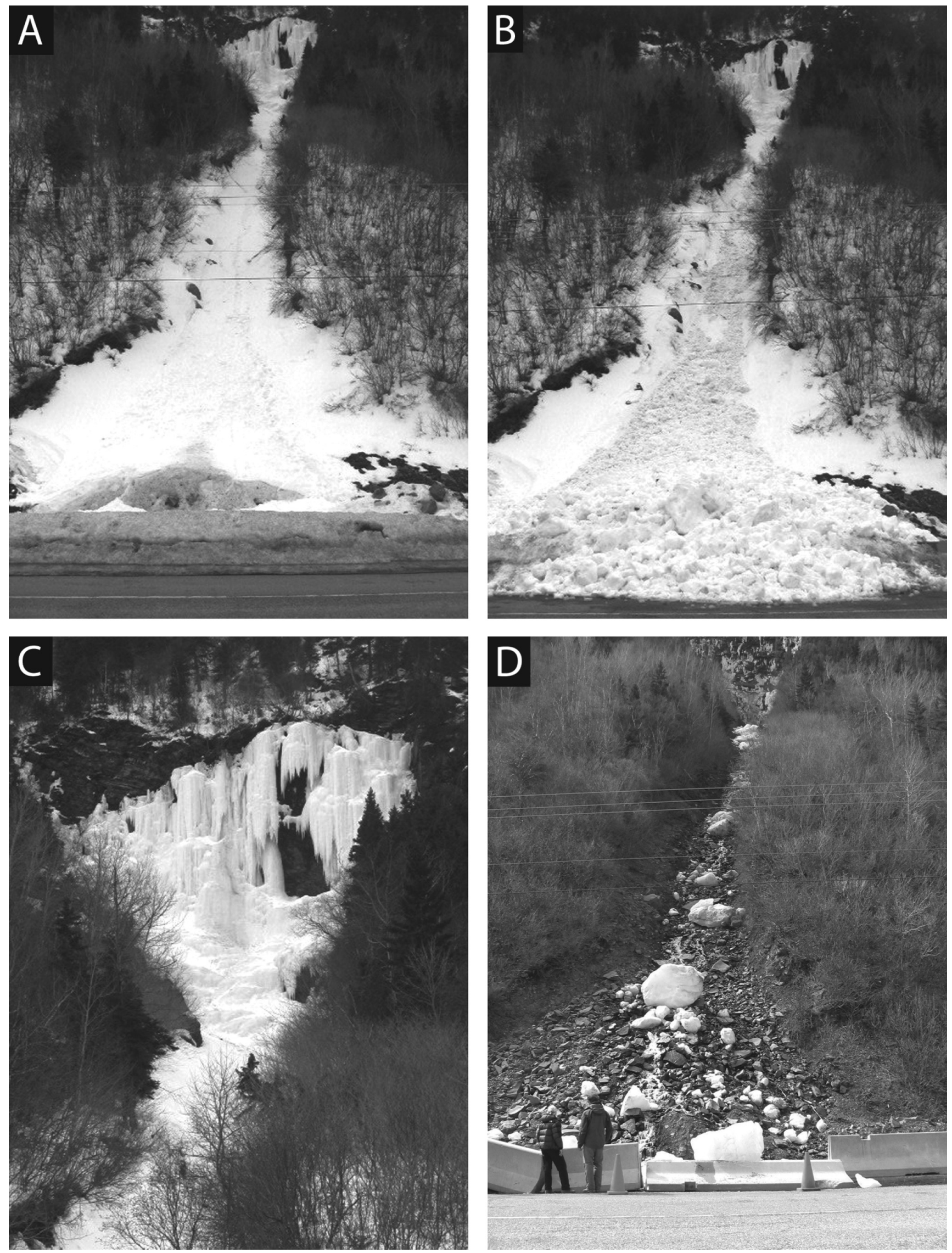

FIGURE 4. (A) Couloir d'avalanche dominé par une carapace de glace près de La Martre. (B) Avalanche provoqué par la chute d'une bloc de glace, le 31 mars 2006. (C) Gros plan de la paroi avant la chute du bloc de glace (comparer les photographies A, B et C). (D) Chute des derniers blocs de glace vers la fin du mois d'avril 2006.

(A) Avalanche path dominated by an ice wall near La Martre. (B) Snow avalanche caused by an ice-block fall, on March 31'st, 2006. (C) Closeup of the ice wall just before the ice-block fall (compare photographs $A, B$, and C). (D) Fall of the last ice blocks in late April 2006. 
(1) malgré des températures moyennes mensuelles bien en deçà de $0{ }^{\circ} \mathrm{C}$, les mois de décembre, janvier, février et mars reçoivent en moyenne entre 1,8 et $16,4 \mathrm{~mm}$ de pluie tombée lors de redoux, phénomène fréquent sous ce climat à tendance maritime (Gagnon, 1970; Environnement Canada, 2005b). (2) La Gaspésie connaît régulièrement d'importantes chutes de neige. On compte en moyenne 8,9 jours par an avec des précipitations neigeuses égales ou supérieures à $10 \mathrm{~cm}$ et 0,84 jour avec une chute de neige égale ou supérieure à $25 \mathrm{~cm}$ (Environnement Canada, 2005b). (3) Avec des températures quotidiennes oscillant de part et d'autre de $0{ }^{\circ} \mathrm{C}$ et des précipitations tombant alternativement sous forme de pluie ou de neige, ce sont les mois de novembre et d'avril qui présentent les conditions météorologiques les plus contrastées (tabl. II).

La région côtière nord-gaspésienne est une région très ventée, d'abord en raison de la fréquence élevée des vents, mais aussi par leur intensité (Hétu, 1992; Environnement Canada, 2006). Les talus d'éboulis côtiers y sont tout particulièrement exposés, spécialement en hiver (Hétu et Vandelac, 1989). Les vents, qui soufflent fréquemment en rafales, peuvent dépasser les $100 \mathrm{~km} / \mathrm{h}$ lors des blizzards, entraînant une déflation éolienne très intense sur les talus d'éboulis. La neige est alors soufflée vers les forêts adjacentes, ce qui entraîne la mise à nu des talus, qui peuvent se dénuder à plusieurs reprises au cours de l'hiver (Hétu et Vandelac, 1989). En revanche, les apports externes sont très limités. L'un des principaux aspects à retenir en ce qui concerne le régime d'enneigement des talus d'éboulis est l'absence d'apports nivéo-éoliens depuis le plateau boisé vers les versants avalancheux. Ceci a trois conséquences: les avalanches sont alimentées par la neige provenant directement des précipitations, les talus d'éboulis se dénudent rapidement après les tempêtes et, contrairement aux milieux alpins, il ne se forme jamais de corniche au sommet des parois (Girard et Hétu, 1989). Ce régime d'enneigement propre au milieu côtier n'est pas sans influencer la dynamique des avalanches.

\section{SOURCES DES DONNÉES ET MÉTHODOLOGIE}

Dans cet article, nous analysons deux séries de données, soit, dans un premier temps, les résultats d'un inventaire de l'activité avalancheuse réalisé par le Ministère des Transports du Québec entre 1987 et 1993 et, dans un deuxième temps, une série d'épisodes avalancheux ponctuels ayant causé des accidents routiers, dont trois mortels.

\section{INVENTAIRE DU MINISTÈRE DES TRANSPORTS DU QUÉBEC}

Étant dominées par des versants raides de forte dénivelée (fig. 2, 3 et 4), les routes du nord de la Gaspésie sont particulièrement exposées à divers mouvements de terrain (Girard et Hétu, 1994; Hétu et al., 1994). Des matériaux en provenance des versants parviennent fréquemment jusqu'au réseau routier (éboulements massifs, blocs rocheux isolés, blocs de glace, avalanches, coulées de débris, glissements pelliculaires), causant des accidents ou encore des interruptions de la circulation routière (fig. 5). Devant l'ampleur du problème, le Ministère des Transports du Québec a lancé en 1987 un programme d'inventaire des mouvements de masse affectant les routes nationales 132 et 198 (fig. 2B). En mai 1987, une unité spéciale appelée Patrouille de Roches a donc été créée. Cette patrouille qui circule à l'année longue, le jour comme la nuit, selon des quarts de huit heures, a pour mandat de localiser et de décrire les débris qui parviennent sur les routes et, le cas échéant, de voir à ce qu'elles soient dégagées dans les meilleurs délais. Afin de faciliter l'archivage des données, la zone d'inventaire a été sub-divisée en 12 secteurs numérotés dont la longueur varie entre 4,1 et $11,8 \mathrm{~km}$, avec une moyenne de $8,8 \mathrm{~km}$ (fig. 2A). Les membres de la patrouille produisent une fiche à chaque fois que le dégagement de la route nécessite l'intervention d'un équipement mécanique. Cette fiche précise le numéro du secteur où le mouvement de masse s'est produit, la nature des matériaux mobilisés (roc, terre, blocs de glace, avalanche), le nombre et la grosseur des blocs de pierre ou de glace, la date et l'heure des observations et une estimation de l'heure du mouvement de masse. Dans le cas des avalanches, les fiches ne contiennent aucune information concernant le type d'avalanche, le volume de neige mobilisé et la distance parcourue. Les seules certitudes que nous avons sont que les avalanches inventoriées par le Ministère des Transports sont des avalanches naturelles et que les masses de neige ont bloqué partiellement ou totalement la route. Les petites avalanches qui n'atteignent pas la chaussée ou l'accotement

TABLEAU II

Normales climatiques de la station de Cap-Madeleine pour la période 1971-2000 (source des données météorologiques: Environnement Canada, 2005b)

\begin{tabular}{|c|c|c|c|c|c|c|c|c|}
\hline Paramètres & Oct. & Nov. & Déc. & Janv. & Fév. & Mars & Avril & Mai \\
\hline Température maximale quotidienne $\left({ }^{\circ} \mathrm{C}\right)$ & 8,8 & 2,7 & $-3,6$ & $-7,2$ & $-6,1$ & $-0,7$ & 4,8 & 11,6 \\
\hline Température minimale quotidienne $\left({ }^{\circ} \mathrm{C}\right)$ & 1,9 & $-3,2$ & $-10,5$ & $-15,6$ & $-14,4$ & $-9,0$ & $-2,1$ & 3,2 \\
\hline Chutes de pluie (mm) & 70,0 & 29,5 & 16,4 & 5,8 & 1,8 & 6,7 & 30,2 & 58,2 \\
\hline Chutes de neige $(\mathrm{cm})$ & 2,4 & 33,0 & 68,9 & 51,1 & 45,3 & 57,3 & 26,4 & 1,4 \\
\hline Jours avec température maximale $>0{ }^{\circ} \mathrm{C}$ & 30,6 & 20,1 & 7,2 & 3,6 & 4,2 & 12,9 & 26,0 & 31 \\
\hline Jours avec pluie $>5 \mathrm{~mm}$ & 4,3 & 2,0 & 1,1 & 0,5 & 0,04 & 0,5 & 1,9 & 3,8 \\
\hline Jours avec pluie $>10 \mathrm{~mm}$ & 2 & 0,78 & 0,62 & 0,21 & 0 & 0,13 & 0,75 & 1,8 \\
\hline Jours avec neige $>10 \mathrm{~cm}$ & 0,13 & 1,0 & 1,9 & 1,5 & 1,5 & 2,1 & 0,83 & 0 \\
\hline Jours avec neige $>25 \mathrm{~cm}$ & 0 & 0,17 & 0,24 & 0,13 & 0 & 0,17 & 0,13 & 0 \\
\hline
\end{tabular}



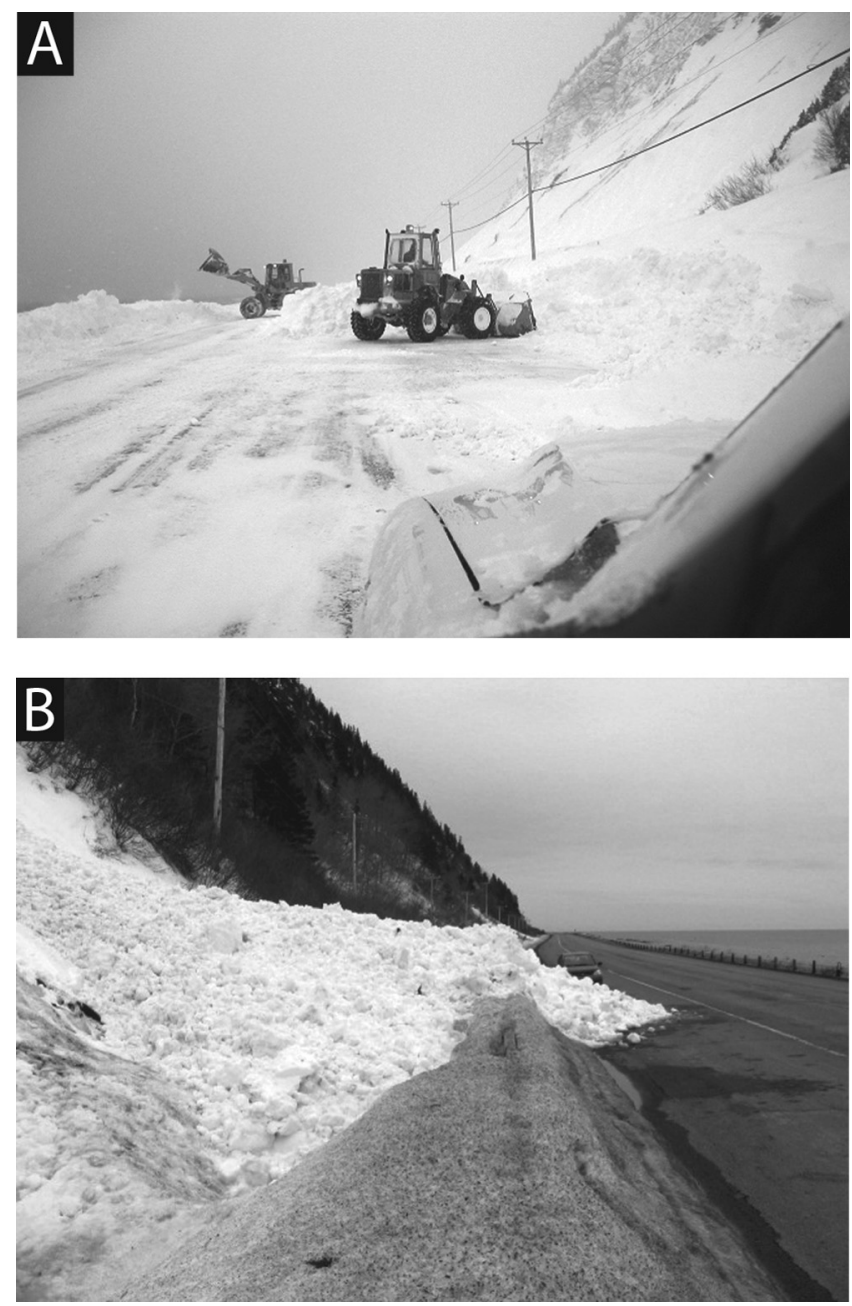

FIGURE 5. (A) Le 30 décembre 2005, deux tracteurs à benne s'affairent à déblayer un dépôt d'avalanche qui obstrue la route nationale 132 à l'ouest de Mont-Saint-Pierre (secteur 100) (photographie: Ministère des Transports du Québec). (B) Dépôt d'avalanche recouvrant l'accotement de la route nationale 132 près de La Martre (secteur 60), le 31 mars 2006.

(A) On December 30 th 2005 , two bulldozers clear up an avalanche deposit obstructing the national road 132 west of Mont-Saint-Pierre (section 100). (Photograph: Ministère des Transports du Québec). (B) Avalanche deposit covering the shoulder of the national road 132 near La Martre (section 60), on March 31st, 2006.

échappent à cet inventaire (Hétu et Gray, 1980 ; Hétu et Vandelac, 1989; Hétu, 1991). L'inventaire est donc axé sur les grosses avalanches à basse fréquence. Dans les secteurs 10, 100, 120 et 130, les avalanches se produisent principalement sur des talus d'éboulis, alors que dans les autres secteurs les avalanches sont confinées aux couloirs à carapace de glace.

Nos propres observations et les quelques photographies Polaroid qui accompagnent les fiches du Ministère indiquent que la longueur habituelle des plus grosses avalanches celles qui atteignent les routes — est de l'ordre de 50 à 250 m dans le cas de la route nationale 132 et de $100 \mathrm{~m}$ à un plus de $300 \mathrm{~m}$ dans le cas de la route nationale 198. Quant aux volumes de neige mobilisés, ils sont estimés entre moins de $200 \mathrm{~m}^{3}$ et plus de $5000 \mathrm{~m}^{3}$. Certaines avalanches peuvent ensevelir une voiture, détruire un camion ou raser un pan de forêt de quelques centaines de mètres carrés, comme ce fut le cas sur l'un des talus d'éboulis de la vallée de Mont-SaintPierre au cours de l'hiver 1995-1996 (Dubé et al., 2004). Sur l'échelle proposée par l'Association Canadienne des Avalanches, l'intensité des avalanches dont il est question ici varie de 1,5 à 3 (Jamieson et Geldsetzer, 1997).

Entre le $1^{\text {er }}$ octobre 1987 et le 31 mai 1993, 19 avalanches ont été inventoriées par la Patrouille de Roches (fig. 6). L'analyse des conditions météorologiques qui prévalaient dans les 15 jours précédant les événements a permis de mieux comprendre les conditions propices au déclenchement des grosses avalanches qui surviennent dans la zone littorale et les basses vallées montagnardes du nord de la Gaspésie. Une attention particulière a été accordée à l'évolution des conditions météorologiques au cours des 48 à 72 heures précédant les avalanches. Les données météorologiques proviennent des stations de Cap-Madeleine ou de Mont-Louis, selon la disponibilité des données. Ces stations sont situées respectivement dans les secteurs 110 et 140 (fig. 2A). Le tableau I présente le total des précipitations neigeuses à CapMadeleine pour chacun des six hivers considérés. Soulignons que quatre des six hivers se situent sous la normale, qui est de $284 \mathrm{~cm}(1971-2000)$.

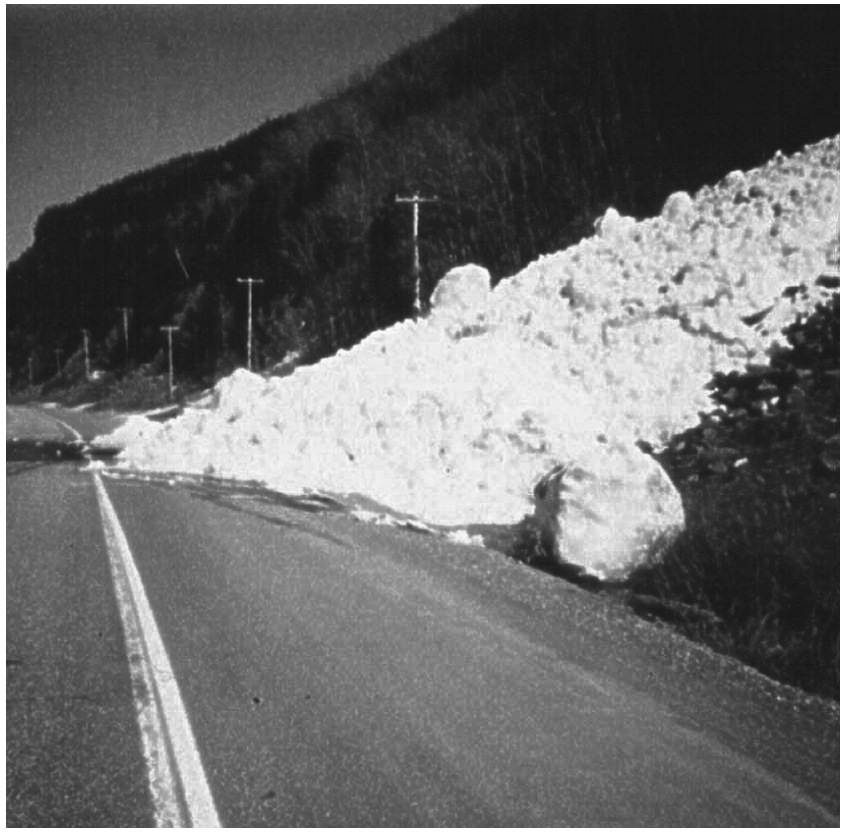

FIGURE 6. Le 24 avril 1991, une avalanche contenant des blocs de glace obstrue partiellement la route nationale 132 près de La Martre (secteur 60) (photographie: Ministère des Transports du Québec).

On April 24 $4^{\text {th }}, 1991$, an avalanche deposit containing several ice blocks recovers half of the national road 132 near La Martre (section 60) (Photograph: Ministère des Transports du Québec). 


\section{ACCIDENTS ROUTIERS PROVOQUÉS PAR DES AVALANCHES}

Les informations concernant les accidents routiers mentionnés dans cet article proviennent de l'analyse croisée des rapports d'enquête des coroners, des articles parus dans les journaux régionaux et des observations compilées par le Ministère des Transports du Québec (bureau de Sainte-Annedes-Monts), auxquelles s'ajoutent les témoignages de cinq résidents des villages de Mont-Louis et de Mont-Saint-Pierre. Le contexte météorologique entourant ces avalanches a pu être reconstitué grâce aux données d'Environnement Canada (2005a) et aux informations publiées dans les journaux.

\section{RÉSULTATS}

\section{INVENTAIRE DU MINISTÈRE DES TRANSPORTS}

Entre le $1^{\text {er }}$ octobre 1987 et le 31 mai 1993, la Patrouille de Roches a noté 15 journées avalancheuses, pour un total de 19 avalanches.

\section{Répartition spatiale: les zones à risque}

Ces 19 avalanches ont affecté six des 12 secteurs d'inventaire (tabl. III). Ce sont, par ordre d'importance, les secteurs $10,130,100$ et 120 qui présentent le plus grand risque, avec respectivement 6,5 , 3 et 3 avalanches. C'est le secteur 10, un talus d'éboulis à exposition OSO - donc très ensoleillé en après-midi - qui est le plus actif. À cet endroit, la route nationale 198 est coincée entre le pied du talus d'éboulis et le lac de l'Anse-Pleureuse (fig. $2 \mathrm{~B}$ et $3 \mathrm{C}$ ). La zone à risque s'étire sur près de deux kilomètres (Transports Québec, 2005). Dans les secteurs 100, 120 et 130, les avalanches prennent naissance sur les talus d'éboulis à exposition nord qui dominent la route nationale 132 (fig. $3 \mathrm{~A}$ et $3 \mathrm{~B}$ ). Ces talus en pente raide (entre 35 et $42^{\circ}$ ) et dont la longueur varie entre 100 et $250 \mathrm{~m}$ se déploient sur plusieurs kilomètres le long de la route. Or, à l'intérieur de ces milieux non confinés que sont les talus d'éboulis, les avalanches sont susceptibles de se produire à n'importe quel endroit d'une fois à l'autre, ce qui complique la gestion du risque. Les avalanches qui se produisent dans les secteurs 60 et 70 (une avalanche chacun) sont, elles, entièrement confinées: elles démarrent dans les étroits couloirs à carapace de glace qui fragmentent localement la forêt (fig. 4). Dans les secteurs $50,80,90,110,140$ et 150 , le risque paraît plutôt modéré puisque aucune avalanche n'a été rapportée durant la période considérée (du $1^{\text {er }}$ octobre 1987 au 31 mai 1993).

\section{Répartition chronologique}

Le tableau III fournit la date des journées avalancheuses pour chacune des six années considérées ainsi que l'heure où les coulées furent découvertes. Quatre points se dégagent de ce tableau:

\section{TABLEAU III}

Avalanches ayant touché les routes nationales 132 et 198 entre le 1er octobre 1987 et le 31 mai 1993 et résumé des conditions météorologiques à Mont-Louis durant les 48 heures précédant les avalanches (sources: Ministère des Transports du Québec et Environnement Canada, 2005a)

\begin{tabular}{|c|c|c|c|c|c|c|c|c|}
\hline \multirow[b]{2}{*}{ Hiver } & \multirow[b]{2}{*}{ Date } & \multirow[b]{2}{*}{ Heure } & \multirow[b]{2}{*}{ Secteur } & \multirow{2}{*}{$\begin{array}{c}\text { Débris } \\
P: \text { pierre } \\
G: \text { glace }\end{array}$} & \multicolumn{4}{|c|}{ Codes Météo* } \\
\hline & & & & & 1 & 2 & 3 & 4 \\
\hline \multirow{8}{*}{$1987-1988$} & 26-01-1988 & $17: 00$ & 130 & & $x$ & $x$ & & \\
\hline & 26-01-1988 & $20: 00$ & 100 & & $x$ & $x$ & & \\
\hline & $26-01-1988$ & $21: 00$ & 10 & & $x$ & $x$ & & \\
\hline & 13-03-1988 & $21: 00$ & 130 & $\mathrm{P}$ & & $x$ & $x$ & \\
\hline & 27-03-1988 & $6: 00$ & 100 & & & & $x$ & \\
\hline & 04-04-1988 & $5: 30$ & 130 & & & & $x$ & \\
\hline & 23-04-1988 & $14: 00$ & 10 & & & $x$ & $x$ & \\
\hline & 23-04-1988 & $16: 10$ & 10 & $\mathrm{P}$ & & $x$ & $x$ & \\
\hline \multirow[t]{4}{*}{$1988-1989$} & 08-04-1989 & $4: 30$ & 10 & $\mathrm{P}$ & $x$ & & $x$ & \\
\hline & 19-04-1989 & $19: 30$ & 120 & & & & $x$ & \\
\hline & 03-05-1989 & $10: 00$ & 100 & $\mathrm{G}$ & & & $x$ & $x$ \\
\hline & 08-05-1989 & $3: 45$ & 120 & & & & $x$ & $x$ \\
\hline \multirow[t]{3}{*}{$1990-1991$} & $12-04-1991$ & $18: 00$ & 120 & $\mathrm{G}$ & & $x$ & $x$ & \\
\hline & $22-04-1991$ & $2: 30$ & 70 & & & & $x$ & $x$ \\
\hline & 24-04-1991 & $14: 15$ & 60 & $\mathrm{G}$ & & & $x$ & \\
\hline \multirow[t]{2}{*}{ 1991-1992 } & 03-05-1992 & $10: 00$ & 10 & & $x$ & & $x$ & $\mathrm{x}$ \\
\hline & 03-05-1992 & $2: 00$ & 130 & & $x$ & & $x$ & $x$ \\
\hline \multirow{2}{*}{ 1992-1993 } & 16-04-1993 & $6: 30$ & 10 & $\mathrm{G}$ & & $x$ & $x$ & $\mathrm{x}$ \\
\hline & 20-04-1993 & $0: 30$ & 130 & $\mathrm{P}$ & & & $x$ & $x$ \\
\hline Nombre de & avalancheu & & & & 3 & 5 & 14 & 6 \\
\hline
\end{tabular}

* Liste des codes météo: 1 - neige la journée même, 2 - neige dans les 48 heures précédant l'avalanche, 3 - température maximale positive la journée de l'avalanche, 4 - pluie la journée de l'avalanche. 
(1) Ce qui frappe d'abord, c'est la distribution inégale des avalanches d'une année à l'autre: la fréquence annuelle va de zéro à huit. Les hivers peuvent à cet égard être regroupés en trois catégories. À l'hiver 1987-1988, très actif, qui rassemble à lui seul $40 \%$ des avalanches (8/19), s'opposent des hivers où l'activité avalancheuse peut être qualifiée de modérée (2 à 4 avalanches: 1988-1989, 1990-1991, 1991-1992, 1992-1993) à nulle (aucune avalanche: 1989-1990). Il est intéressant de souligner que l'hiver le plus actif (1987-1988) est celui qui a connu le plus grand nombre de tempêtes (tabl. I).

(2) Parmi les 15 journées avalancheuses inventoriées, trois se distinguent pour avoir produit chacune de 2 à 3 avalanches. II s'agit du 26 janvier et du 23 mars 1988 ainsi que du 3 mai 1992. Les 12 autres journées de l'inventaire n'ont produit qu'une seule avalanche chacune.

(3) Les grosses avalanches qui sont parvenues jusqu'aux routes constituent, dans le cadre de cet inventaire, un phénomène essentiellement printanier puisque 14 des 19 avalanches inventoriées se sont produites en avril (10) et en mai (4). Quatre d'entre elles contenaient des blocs de glace (fig. 6).

(4) Sur ces versants nord peu ensoleillés, les avalanches surviennent aussi bien le jour que la nuit.

\section{Rareté des grosses avalanches chargées}

L’impact géomorphologique des avalanches qui parviennent jusqu'aux routes semble plutôt modéré, car seulement cinq d'entre elles transportaient des débris rocheux. II semble que la plupart des grosses avalanches ne mobilisent que les couches superficielles du manteau neigeux, sans toucher au substrat minéral. À cet égard, elles semblent très différentes des petites avalanches très chargées en débris qui ont été observées à maintes reprises sur les mêmes talus (Hétu et Vandelac, 1989; Hétu, 1991, 1995).

\section{Déclenchement des avalanches}

Le tableau III résume les conditions météorologiques prévalant dans les 48 heures précédant les 15 journées avalancheuses rapportées tandis que la figure 7 illustre l'évolution à moyen terme des conditions météorologiques pour un certain nombre de journées avalancheuses représentatives des situations rencontrées. D'abord ce qui frappe, c'est le rôle déterminant joué par la fonte printanière et la pluie: 14 des 15 journées avalancheuses surviennent alors que les températures maximales quotidiennes sont positives; près de la moitié (6/15) correspondent à des journées pluvieuses. Le rôle de la pluie s'avère plus important encore si l'on étend l'analyse aux dernières 48 heures puisque le nombre de journées avalancheuses concernées grimpe alors à 12. Par exemple, le 19 avril 1989, il était tombé $4 \mathrm{~mm}$ de pluie la veille de l'avalanche (fig. 7A). Les deux avalanches du 23 avril 1988 surviennent après deux journées très arrosées qui totalisaient $44,2 \mathrm{~mm}$ de pluie (39 mm, le 21 avril et 5,2 mm, le 22 avril).

Les grosses chutes de neige suivies ou non par de la pluie constituent également un important facteur de déclenchement. La moitié des avalanches (11/19) coïncident avec une chute de neige tombée le jour même (3/19), la veille (5/19) ou les deux (3/19), pour un total de sept journées avalancheuses sur 15 (tabl. III). La journée avalancheuse qui a produit le plus grand nombre de décrochements (26 janvier 1988: 3 avalanches) coïncide avec une grosse tempête au cours de laquelle il est tombé $60 \mathrm{~cm}$ de nouvelle neige en deux jours, dont $50 \mathrm{~cm}$ le jour même des avalanches (fig. 7B). C'est la seule journée avalancheuse de l'inventaire qui survient alors que les températures sont négatives. Nous en déduisons qu'il s'agissait probablement de grandes avalanches de plaques de neige sèche. Deux autres journées avalancheuses se signalent par des chutes de neige relativement importantes, soit le 8 avril 1989 (neige: $16 \mathrm{~cm}$, une avalanche, fig. 7A) et le 3 mai 1992 (neige: $5 \mathrm{~cm}$, pluie: $7,9 \mathrm{~mm}, 2$ avalanches). Dans les trois autres cas où des chutes de neige fraîche sont impliquées, les avalanches se sont produites en différé, c'est-à-dire le lendemain (13 mars 1988, 12 avril 1991) ou le surlendemain (23 avril 1988), et ce, avec le concours de la pluie dans deux des trois cas (23 avril 1988, 12 avril 1991). II n'est pas rare dans le contexte gaspésien que l'on passe de la neige à la pluie au cours de la même précipitation. Une telle situation peut également provoquer des coulées de neige liquéfiée (slushflow) comme ce fut le cas le 18 mars 1980 à Mont-Saint-Pierre (Hétu et Vandelac, 1989).

Quatre des 19 avalanches inventoriées contenaient des blocs de glace (fig. 6). Ces quatre avalanches se sont produites en avril et mai, alors que les températures maximales quotidiennes étaient largement positives. Cette période correspond précisément à l'écroulement des carapaces de glace (fig. 4D). Les blocs de glace libérés font couramment de 1 à $4 \mathrm{~m}^{3}$ et les plus gros approchent les $10 \mathrm{~m}^{3}$. II est fort probable que les quatre avalanches printanières dont il est question ici ont été déclenchées par la chute de blocs de glace sur un manteau neigeux que la fonte avait rendu instable. Cette hypothèse a pu être attestée le 31 mars 2006 dans un couloir situé le long de la route nationale 132 à l'ouest de La Martre. Le bloc de glace qui s'est détaché du sommet de la paroi ce jour-là faisait environ $9 \mathrm{~m}^{3}$ (fig. 4A, B et C); il a provoqué une grosse avalanche de neige humide qui s'est arrêtée sur l'accotement (fig. 5B).

Même si les vents sont presque toujours présents dans cet environnement maritime, il n'est pas certain que les surcharges éoliennes constituent un facteur de déclenchement efficace dans le contexte précis de cet inventaire, dans la mesure où la plupart des journées avalancheuses (14/15) correspondent à des périodes de redoux accompagnées de pluie. Or la neige humide est peu mobile. En outre, dans ce milieu essentiellement forestier, où les apports nivéo-éoliens externes sont quasi-impossibles, le vent a plutôt tendance à dénuder les talus, ce qui contribue à réduire les risques d'avalanche entre les tempêtes.

\section{ACCIDENTS ROUTIERS PROVOQUÉS PAR DES AVALANCHES}

L'analyse des quelques accidents que nous avons pu retracer, dont trois qui se sont avérés mortels, confirme l'importance des tempêtes de neige comme facteur de déclenchement des avalanches sur les talus d'éboulis côtiers. 

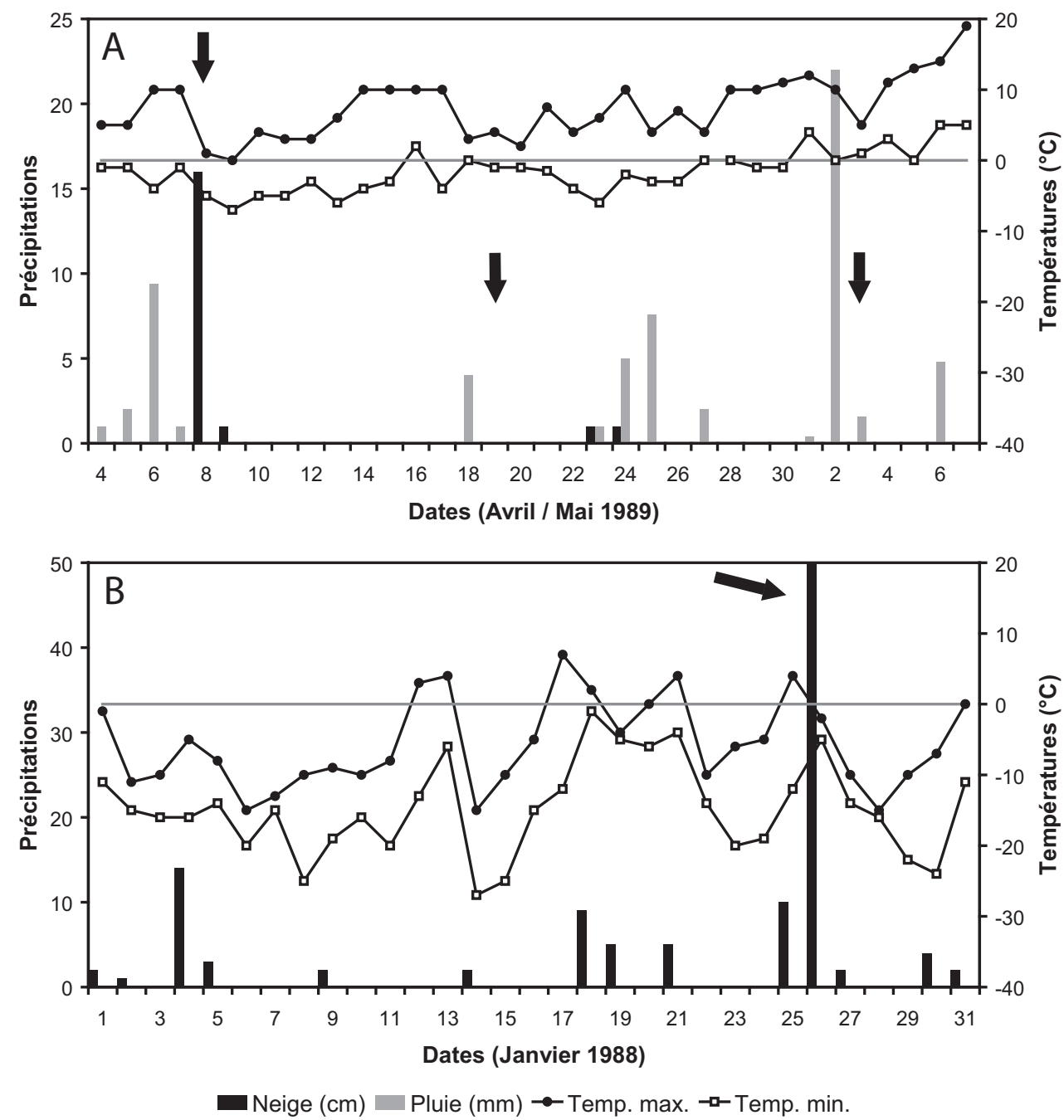

FIGURE 7. (A) Régime avalancheux printanier. Les flèches indiquent les dates des avalanches: 8 avril, 19 avril et 3 mai 1989. (B) Régime avalancheux de tempête. La flèche indique l'avalanche du 26 janvier 1988 (source des données météorologiques: Environnement Canada, 2008, station de Mont-Louis).

(A) Spring avalanche regime. The arrows indicate the avalanche days: April $8^{\text {th }}$, April $19^{\text {th }}$ and May $3^{\text {rd }}$, 1989. (B) Storm avalanche regime. The arrow indicates the avalanche cycle of January 26 ${ }^{\text {th }}, 1988$ (Meteorological data source: Environment Canada, 2008, Mont-Louis station).
Avalanche mortelle du 24 janvier 1935

L'avalanche mortelle la plus ancienne connue à ce jour en Gaspésie est survenue à Mont-Saint-Pierre en janvier 1935. Dans un court article paru le $1^{\text {er }}$ février 1935 dans l'Action catholique (p. 8), on pouvait lire ce qui suit: «Monsieur Georges Cloutier a été tué et trois autres personnes ont vu la mort de près lorsqu'un éboulis de neige les a surpris en cours de route. Monsieur Cloutier était parti pour aller réparer le chemin et il faisait route avec Monsieur Adrien Mercier qui conduisait deux voyageurs revenant des chantiers. Un éboulis se produisit alors que la voiture longeait la falaise et les quatre hommes furent pratiquement ensevelis. Monsieur Cloutier avait succombé lorsqu'on parvint à le dégager». Le rapport du coroner précise que l'accident s'est produit le 24 janvier 1935 à Mont-SaintPierre. II indique que Monsieur Cloutier est mort par asphyxie après avoir séjourné plus d'une heure sous quatre à cinq pieds de neige. Il ajoute qu'un cheval — vraisemblablement celui qui tirait la voiture - a été enseveli en même temps que Monsieur Cloutier. Selon Monsieur Jacques Mercier, rencontré à MontSaint-Pierre le 28 juillet 2006, un autre passager aurait été lui aussi complètement enseveli. Ce dernier aurait été dégagé vivant après avoir séjourné sous la neige pendant quatre heures. II décédera deux ou trois ans plus tard à la suite de problèmes pulmonaires que ses proches attribuent à son séjour prolongé sous la neige. Monsieur Jacques Mercier nous a précisé que l'accident s'était produit à l'ouest de la baie à Mont-Saint-Pierre, près de la chute du Pisseux à François (fig. 8). Ce versant, situé sous le vent par rapport aux vents dominants, accumule généralement beaucoup plus de neige que le talus oriental. II s'agit d'un secteur extrêmement actif qui produit régulièrement des avalanches. Les photographies de l'époque montrent que la route était alors située juste au pied des talus d'éboulis et donc directement exposée aux avalanches (fig. 8). L'avalanche du 24 janvier 1935 coïncide avec une grosse tempête de neige; il est tombé ce jour-là à MontLouis 35,6 cm de neige (Environnement Canada, 2005a).

\section{Avalanches de l'hiver 1955-1956}

Le 25 janvier 1956, les avalanches firent une deuxième victime à Mont-Saint-Pierre. L'édition du 2 février 1956 de $\mathrm{La}$ Voix de Matane (p. 1) précise les circonstances de l'accident: «Un jeune homme [...] âgé de 18 ans, de Mont-Saint-Pierre, 


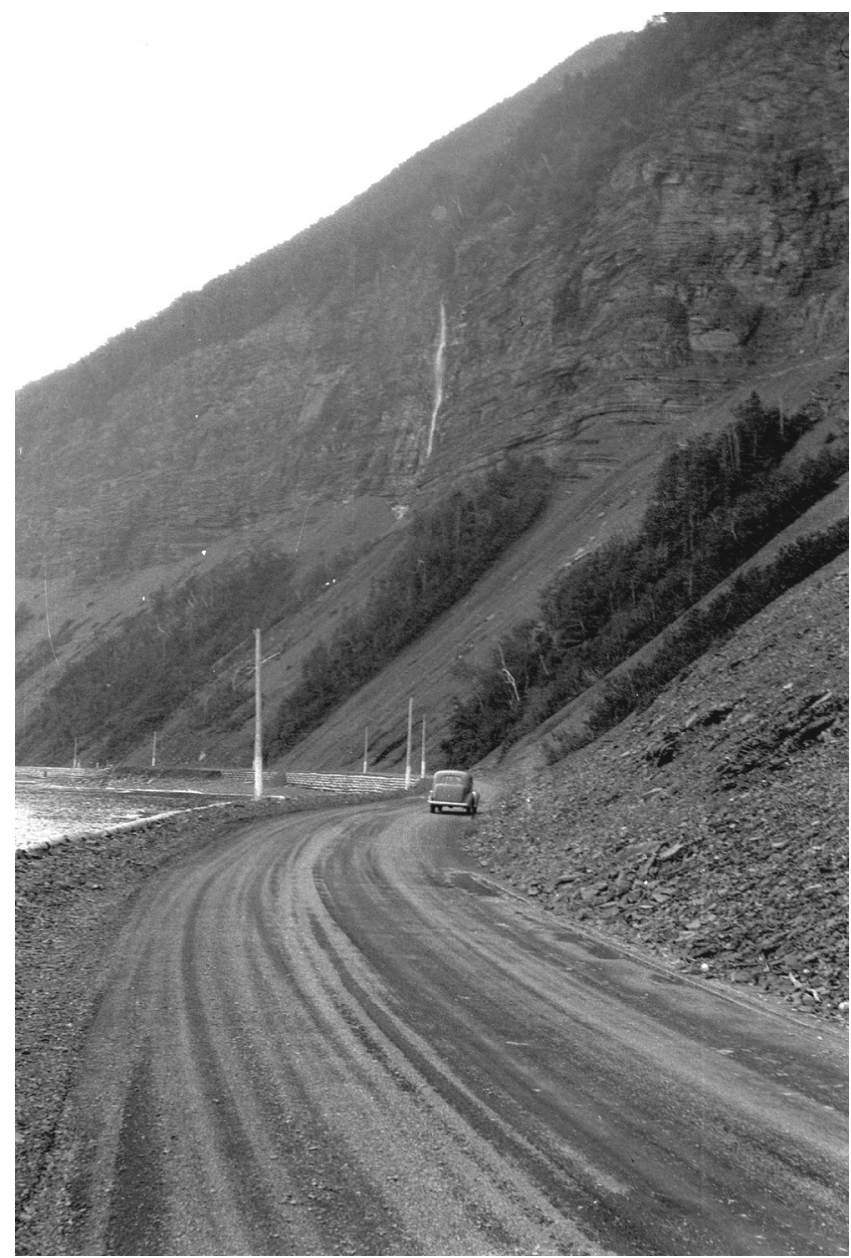

FIGURE 8. Les talus d'éboulis de la baie de Mont-Saint-Pierre (secteur 100) en 1942. À cette époque, la route était située juste au pied des pentes avalancheuses. On aperçoit au centre la chute du Pisseux à François (photographie: Archives nationales du Québec).

Scree-slopes near Mount-Saint-Pierre (sector 100) in 1942. At that time, the road was located just at the foot of the avalanche slopes. One sees in the centre the waterfall called Pisseux à François (Photograph: Archives Nationales du Québec).

a perdu la vie alors qu'il participait aux travaux de déblaiement d'un éboulis de neige qui venait de se produire sur une route de cette localité. Une nouvelle avalanche se produisit qui ensevelit le jeune homme sous plusieurs pieds de neige. Les secouristes durent pelleter cinq heures avant de découvrir le corps sans vie de la victime». Cet accident est survenu au pied du talus d'éboulis qui longe la baie de Mont-SaintPierre à l'ouest du village (fig. 8). D'après l'article cité ci-dessus, il semble s'être produit deux avalanches au même endroit, et ce, à quelques minutes d'intervalle, ce qui traduit une grande instabilité du manteau neigeux. Cet accident est la conséquence d'une séquence météorologique exceptionnelle. En janvier 1956, le total des chutes de neige à CapMadeleine a atteint le chiffre record de $270,5 \mathrm{~cm}$ (Environnement Canada, 2005a). II s'agit de la plus importante précipitation mensuelle depuis la fin du $19^{e}$ siècle (fig. 9).

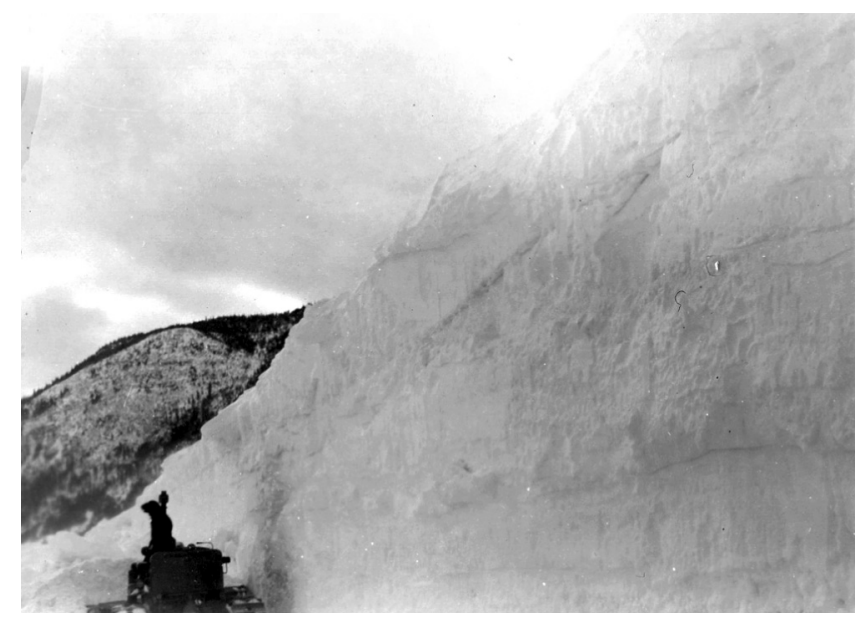

FIGURE 9. L'hiver 1955-1956 se distingue par des chutes de neige bien supérieure à la normale. Une déneigeuse à l'œuvre près du pont de l'Anse-Pleureuse (route nationale 132) à la fin de janvier 1956 (photographie: Ministère des Transports du Québec).

The winter 1955-1956 is characterized by snowfalls quite higher than the normal. Snow removal near the bridge of Anse-Pleureuse (national road 132) in late January 1956 (Photograph: Ministère des Transports du Québec).

La figure 10 montre la répartition quotidienne des précipitations. L'avalanche mortelle du 25 janvier survient après 11 jours de précipitations ininterrompues au cours desquels il est tombé 213,6 cm de neige, dont 30,5 cm le jour de l'avalanche. Cette longue précipitation a provoqué plusieurs avalanches dans au moins trois secteurs distincts: près de Mont-SaintPierre (route nationale 132, secteur 100), entre l'AnsePleureuse et Gros-Morne (route nationale 132, secteur 120), entre l'Anse-Pleureuse et Murdochville (route nationale 198, secteur 10).

II semble que les pentes sur-enneigées de la région soient demeurées instables une grande partie de l'hiver. La Voix Gaspésienne du 24 mars 1956 rapporte en effet un second épisode avalancheux qui a provoqué lui aussi un accident routier: "Le 9 mars dernier [sic] une forte tempête de neige s'est abattue sur Marsoui et les environs. La charrue à neige [...] a été engloutie par un éboulis [sic] à Mont St-Pierre. La charrue, ayant perdu une chaîne, ne pouvait plus avancer, et c'est alors que [les opérateurs] se sont rendus au village de Mont StPierre pour chercher de l'aide. À leur retour vers la charrue, ils ont constaté qu'un éboulis [sic] s'était détaché et que trois pieds de neige recouvrait la cabine du camion". II est clair que les «éboulis » dont il est question ici sont en fait des avalanches. Encore une fois, elles semblent s'être produites sur I'un des deux talus d'éboulis qui encadrent la baie de MontSaint-Pierre (fig. 3B et 8), c'est-à-dire dans le secteur 100 (fig. A) qui apparaît décidément comme l'un des plus actifs de la région. Tout comme à Mont-Saint-Pierre, le 25 janvier de cette même année, il semble bien, à la lecture de cet article, que deux avalanches aient touché le véhicule, une première qui l'a forcé à s'immobiliser après l'avoir endommagé et une seconde qui l'a enfoui après le départ des opérateurs. Cet accident coïncide encore une fois avec une importante tempête: le 8 mars (et non le 9 comme l'indique l'article), les stations de 
Cap-Chat et de Mont-Louis enregistrent respectivement $26,7 \mathrm{~cm}$ et $35,6 \mathrm{~cm}$ de neige (Environnement Canada, 2005a). D'après les données météorologiques, il est clair que la tempête remonte au 8 mars, mais il se peut - comme l'indique $\mathrm{La}$ Voix Gaspésienne - que les avalanches ne se soient produites que le lendemain.

\section{Avalanches de mars 1960}

Un court article paru dans l'édition du jeudi 17 mars 1960 de La Voix Gaspésienne (p. 5) décrit une crise avalancheuse spectaculaire qui semble avoir générée plusieurs avalanches chargées de débris rocheux. "Des éboulis de roc et de neige ont dévalé les flancs abrupts des montagnes qui surplombent la route nationale dans les environs de Mont-Louis la semaine dernière et ont interrompu la circulation pendant quelques jours. Des dommages sérieux ont été causés aux poteaux et aux fils téléphoniques qui se sont renversés ou cassés sous la violence du choc. [...] Les fortes chutes de neige, le temps doux et le vent ont été la cause de ces éboulis". La date exacte de cette crise avalancheuse - qui s'est peut-être déroulée sur quelques jours - n'est pas précisée dans l'article, mais les données météorologiques d'Environnement Canada (2005a) confirment qu'une importante tempête s'est abattue sur la région durant la semaine qui a précédé la parution de l'article. Durant cette tempête qui a duré quatre jours — soit du 10 au 13 mars inclusivement — il est tombé à MontLouis près de $130 \mathrm{~cm}$ de neige. Cette importante surcharge a probablement provoqué des avalanches de fond comme l'indique la présence de débris rocheux dans les dépôts.

\section{Accident mortel du 20 décembre 1971 à Mont-Saint-Pierre}

Vers 15h00, le 20 décembre 1971, Madame Anna Robinson a perdu la maîtrise de son véhicule en tentant de contourner une coulée d'avalanche qui obstruait la route nationale 132 à la hauteur du mont Saint-Pierre, à l'est du village du même nom (Latulippe, 1971). Madame Robinson est morte d'hypothermie après que son automobile eut plongé dans la mer. Monsieur Léon Dugas, qui l'accompagnait, a réussi à s'extraire du véhicule. Nageant à travers les glaces, il a pu rejoindre la rive. Le fils de la victime, Monsieur Jacques Dugas, est formel: l'avalanche, déjà immobile lorsque survint l'accident, n'a pas heurté le véhicule. Cet accident démontre que le risque relié aux avalanches en milieu routier ne concerne pas que les véhicules qui sont directement frappés par l'avalanche (McClung, 1999). Le risque perdure tant que le dépôt de l'avalanche n'a pas été déblayé.

Les événements météorologiques marquants (station de Mont-Louis) ayant conduit au déclenchement de cette avalanche sont, dans l'ordre: (1) une pluie de 10,2 mm tombée le 11 décembre alors que la température maximale quotidienne à Mont-Louis atteignait $+5^{\circ} \mathrm{C}$; (2) cette pluie a été suivie par une importante chute des températures maximales qui sont demeurées négatives à compter du 14 décembre jusqu'au jour de l'avalanche. Cette baisse des températures a probablement provoqué la formation d'une croûte de regel sur la neige qui s'était accumulée jusque-là (il était tombé $55,8 \mathrm{~cm}$ de neige entre le 11 novembre et le 10 décembre). (3) Entre les 13 et 17 décembre inclusivement, la région a reçu plus $40 \mathrm{~cm}$ de nouvelle neige. L'avalanche est survenue trois jours après la fin des précipitations neigeuses, au lendemain d'une nuit relativement froide $\left(-18,9{ }^{\circ} \mathrm{C}\right.$ à Mont-Louis). II s'agit de toute évidence d'une avalanche différée reliée à la métamorphose de la neige. L'accumulation d'une épaisse couche de neige sèche et froide sur une croûte de regel, dans un contexte de température à la baisse, a probablement entraîné la formation d'une couche faible constituée de grains à faces planes (Birkeland, 1998; Colbeck et Jamieson, 2001) qui aura, à terme, favorisé le déclenchement de l'avalanche.

\section{Crise avalancheuse des 2 et 3 février 1980}

Les abondantes chutes de neige qui se sont abattues sur la région de Mont-Louis les $1^{\text {er }}$ et 2 février 1980 (30 cm au total) ont provoqué de nombreuses avalanches près de MontSaint-Pierre (Henley, 1980). Ces avalanches ont obstrué la route nationale 132 sur une longueur de près de $600 \mathrm{~m}$, immobilisant l'ambulance de Mont-Louis pendant plusieurs heures. Le travail de déblaiement fut particulièrement difficile car dès qu'on avait réussi à pratiquer un passage, de nouvelles coulées de neige l'obstruaient à nouveau. Ces avalanches répétées démontrent que le manteau neigeux est demeuré instable pendant plusieurs heures.

\section{DISCUSSION}

\section{ZONES À RISQUE}

Dans les limites du territoire étudié (fig. 2A), les secteurs routiers les plus exposés au risque avalancheux sont, par ordre d'importance décroissante, les talus d'éboulis qui bordent la baie de Mont-Saint-Pierre (secteur 100) où se sont produits les trois accidents routiers mortels connus à ce jour, les talus d'éboulis partiellement boisés qui dominent la route nationale 198 à la hauteur du lac de l'Anse-Pleureuse (secteur 10) et les talus d'éboulis qui longent la route nationale 132 entre l'Anse-Pleureuse et Manche-d'Épée (secteurs 120 et 130). La construction de remparts entre la route et les talus d'éboulis, efficace dans le cas des chutes de blocs rocheux, n'élimine pas le risque avalancheux dans la mesure où souvent la dépression côté versant est remplie de neige. Les avalanches peuvent donc la franchir aisément, à moins de la déblayer régulièrement comme le font les employés du Ministère des Transports dans certains secteurs.

À Mont-Saint-Pierre, le niveau de risque, très élevé au début du $20^{\mathrm{e}}$ siècle, alors que la route nationale 132 était située juste au pied des talus d'éboulis (fig. 8), a quelque peu diminué après la reconfiguration de son tracé au début des années 1980, particulièrement à l'est de la baie où la route est maintenant située à une trentaine de mètres en moyenne du talus. À l'ouest de la baie, en revanche, la route, située par endroits à moins de $15 \mathrm{~m}$ du pied du talus, est encore trop proche du versant. Régulièrement, encore aujourd'hui, des avalanches obstruent la route (fig. 5A), surtout dans le secteur du Pisseux à François qui a fait à ce jour deux victimes. 


\section{DÉCLENCHEMENT DES AVALANCHES}

Il semble, à la lumière des données disponibles, que les versants côtiers connaissent deux principaux régimes avalancheux: un premier, bien marqué, que l'on pourrait qualifier de printanier (fig. 7A) et un second associé aux tempêtes de neige (fig. 7B et 10).

\section{Régime printanier d'avalanches de fonte}

La fonte printanière, la pluie et l'écroulement des carapaces de glace constituent les principaux agents déclencheurs des avalanches qui parviennent jusqu'aux routes de la Gaspésie septentrionale. Le rôle de la pluie et de la fonte printanière comme facteur de déclenchement des avalanches est bien connu et a été souligné à maintes reprises (Ward, 1984; Butler, 1986 ; Conway et Raymond, 1993; Conway, 2004; McClung et Schaerer, 2006). Le rôle des chutes de blocs de glace était pressenti depuis quelques années déjà puisque quelques dépôts d'avalanche contenant des blocs de glace avaient été observés dans la région (fig. 6). II a pu être confirmé de visu le 31 mars 2006 (fig. 4 et 5B). À l'échelle de la région, ce régime dominé par les avalanches humides de printemps est propre aux versants à exposition nord où la neige s'attarde certaines années jusqu'à la fin du mois de mai. Or, à cette période de l'année, les précipitations liquides sont relativement fréquentes (tabl. II).

\section{Régime avalancheux de tempête}

La surcharge engendrée par un apport massif de nouvelle neige lors des fortes précipitations (tempêtes hivernales) représente l'autre facteur de risque. II est bien connu que la probabilité d'avalanche augmente rapidement avec la quantité et l'intensité (taux horaire) des précipitations neigeuses (Perla, 1970 ; Bigras et Hébert, 1982; Ward, 1984 ; Bakkehoi, 1987; Conway et Wilbour, 1999; Höller, 1999; Birkeland et al., 2001 ; Reardon et al., 2004 ; McClung et Schaerer, 2006 ; Jomelli et al., 2007). Les accidents routiers de 1935, 1956, 1960 et 1980, qui coïncident avec des tempêtes hivernales, démontrent que les grosses avalanches qui parviennent jusqu'aux routes gaspésiennes ne sont pas un phénomène strictement printanier. L'importance des grosses chutes de neige comme facteur de déclenchement des avalanches au Québec est confirmée par l'analyse des avalanches mortelles connues depuis 1825 (Hétu et Brown, 2006).

L'importance des fortes précipitations neigeuses comme facteur de déclenchement des avalanches en Haute-Gaspésie avait déjà été évoquée par Dubé et al. (2004). Ceux-ci ont constaté que les années de forte activité avalancheuse, reconstituées à partir de données dendrochronologiques, se signalent par des précipitations neigeuses bien supérieures à la normale, à l'exception de l'hiver 1897-1898 sur lequel nous reviendrons (tabl. I). Nous croyons que la relation notée par Dubé et al. (2004) entre l'activité avalancheuse et le total des précipitations solides hivernales n'est qu'apparente. Elle cache une autre réalité, soit le rôle déterminant des tempêtes. En effet, comme l'ont démontré Loup et Lovie (1966), ce n'est pas tant le total des précipitations hivernales que la répartition des chutes de neige qui fait la différence. Une analyse plus fine des données météorologiques de la station de Cap-Madeleine montre que les années de forte activité avalancheuse identifiées par Dubé et al. (2004) se distinguent (sauf l'hiver 19761977) par un nombre de tempêtes bien supérieur à la normale, en particulier les plus grosses (tabl. I). Vu sous cet angle, le paradoxe soulevé par l'hiver 1897-1898 s'explique aisément. En fait, quant aux tempêtes, il sort lui aussi de l'ordinaire: même si le total des chutes de neige enregistrées durant cet hiver est tout à fait moyen $(289,1 \mathrm{~cm})$, une analyse fine $\mathrm{du}$ régime des précipitations révèle qu'il a connu un nombre de grosses tempêtes $((25 \mathrm{~cm} / \mathrm{j})$ nettement supérieur à la moyenne ( 5 contre 0,84 par hiver en moyenne) qui se rapproche de ce qui a été observé en 1955-1956 (6 tempêtes $>25 \mathrm{~cm}$ de neige en $24 \mathrm{~h}$ ), un hiver qui se démarque par la sévérité des avalanches rapportées dans les journaux (dont une mortelle). Ainsi, la relation mise en évidence par Dubé et al. (2004) serait en bonne partie tautologique dans la mesure où les années qui connaissent de nombreuses tempêtes ont de fortes chances d'obtenir un score élevé au total des précipitations.

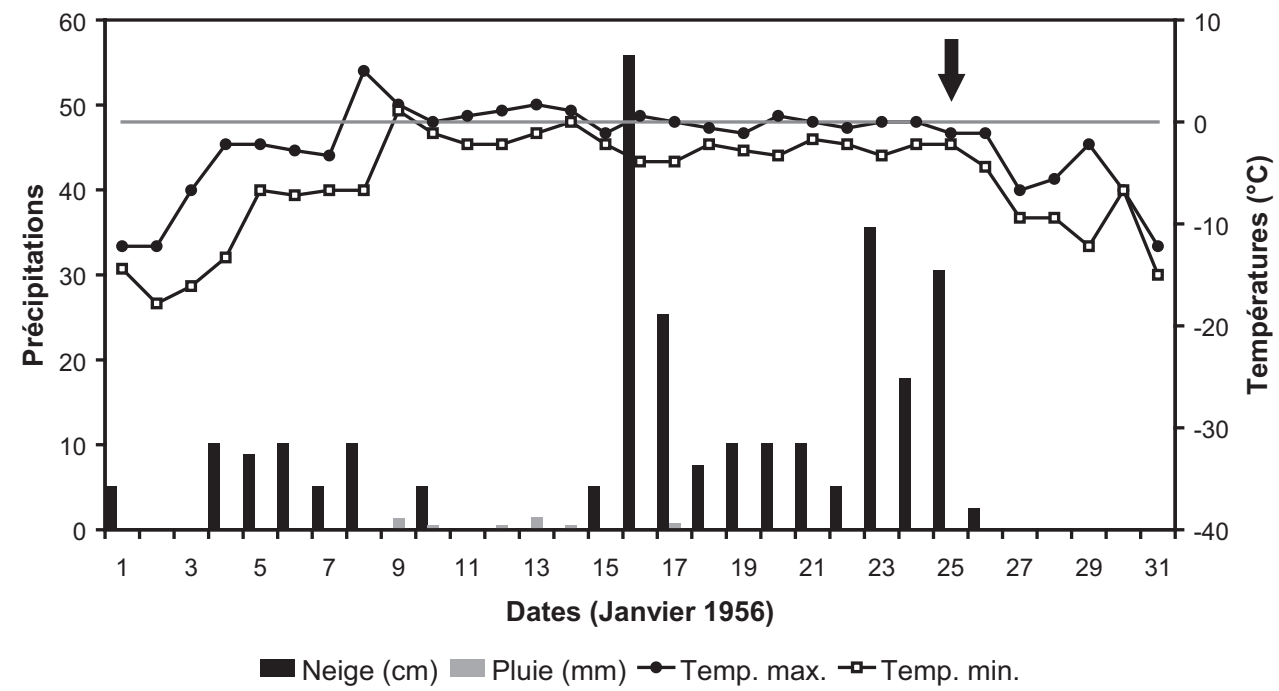

FIGURE 10. Évolution des conditions météorologiques à CapMadeleine en janvier 1956 ; la flèche indique l'avalanche du 25 janvier 1956 à Mont-SaintPierre (source des données météorologiques: Environnement Canada, 2008).

Evolution of meteorological conditions at Cap-Madeleine during January 1956; the arrow indicates the January $25^{\text {th }}$, 1956 avalanche at Mont-Saint-Pierre (Meteorological data source: Environment Canada, 2008). 
Enfin, il est intéressant de souligner que les avalanches de $1935,1960,1971$ et 1980 , dont nous avons retrouvé la trace dans les journaux, ne semblent pas avoir été enregistrées par la végétation. Ces quatre années ne figurent pas dans la chronologie des avalanches élaborée par Dubé et al. (2004) à partir de données dendroécologiques. Pourtant, les épisodes avalancheux de mars 1960 et de février 1980, en particulier, semblent avoir produit de nombreuses coulées de neige dans la région. Les avalanches de l'hiver 1955-1956 ont en revanche laissé de très nombreuses traces, à la fois dans la végétation des trois versants étudiés par Dubé et al. (2004) et dans la mémoire collective des résidents du nord de la Gaspésie (articles de journaux, photographies, témoignages). L'hiver 1955-1956 apparaît, en définitive, comme étant le plus marquant du $20^{\mathrm{e}}$ siècle dans le nord de la Gaspésie en ce qui concerne l'activité avalancheuse.

\section{CONCLUSION}

Les avalanches ont tué en Gaspésie au moins six personnes entre 1935 et 2007 (Hétu et Brown, 2006). Elles représentent un risque notoire tant pour les routes que pour les adeptes de la montagne. La dendrochronologie a permis d'en établir la fréquence minimale à long terme (Hétu, 1986, 1990, 1991, 1995; Hétu et Vandelac, 1989 ; Dubé et al., 2004 ; Germain, 2005; Germain et al., 2005). Elle a également permis de proposer des scénarios avalancheux plausibles qui devront toutefois être validés (Dubé et al., 2004; Germain, 2005). Il est maintenant nécessaire de poursuivre les recherches sur la climatologie de la neige et des avalanches afin de préciser les conditions nivéo-météorologiques propices au déclenchement des coulées de neige dans le contexte spécifique de la Gaspésie, et ce, tant en montagne que dans la zone littorale où sont concentrés les villages et les routes qui les desservent. Cette étude est un premier pas dans cette direction. Les données présentées dans cet article ont permis d'identifier deux scénarios avalancheux: un régime printanier associé à la fonte, à la pluie et au démantèlement des carapaces de glace et un régime hivernal modulé par les tempêtes de neige. Toutefois, les deux régimes évoqués ci-dessus de manière un peu schématique ne doivent pas occulter des situations plus complexes. L'hiver gaspésien se distingue par sa grande variabilité (Hétu et Vandelac, 1989). Des périodes de redoux, souvent accompagnés de pluie, alternent avec des tempêtes de neige et des épisodes de froid intense (tabl. I). Ces conditions changeantes, sur des pas de temps relativement courts, engendrent un manteau neigeux très complexe, comportant de nombreuses croûtes de regel et des couches de glace qui alternent avec des couches de neige de faible densité, déposées par temps calme, et des plaques de neige soufflée beaucoup plus compactes. Dans ces conditions changeantes, différents types de métamorphoses génératrices de couches fragiles, comme par exemple l'apparition de cristaux à faces placés au-dessus d'une croûte de regel enfouie (Colbeck et Jamieson, 2001) ou encore le développement de givre de profondeur (Ancey, 1996; McClung et Schaerer, 2006), sont susceptibles de se produire. II reste à préciser le rôle joué par ces différents scénarios dans le déclenchement des avalanches naturelles qui frappent les routes du nord de la Gaspésie.

\section{REMERCIEMENTS}

Plusieurs personnes nous ont aidé à rassembler, à traiter et à analyser l'information présentée dans cette publication: le personnel du bureau du Ministère des Transports du Québec à Sainte-Anne-des-Monts, en particulier Guy St-Pierre et Alain Dumont ; Jean-Pierre Gagnon et Dominic Boucher du Centre d'avalanche de la Haute-Gaspésie; Louis Gasse, Jacques Dugas, Jacques Mercier, Omer Cloutier et Renaud Coulombe, résidents de Mont-Saint-Pierre et de Mont-Louis; Kati Brown, Samuel Bolduc, Catherine Plante, Stéphanie Friesinger, Gaétan Guay-Tanguay, Jean-François Girard (actuellement professeur au Collège de Rimouski), Suzanne Gagnon (cartographie) et Pierre Collins (archives) de l'Université du Québec à Rimouski. Ces recherches ont été supportées par le Conseil de Recherches en Sciences Naturelles et en Génie du Canada (CRSNG), Hydro-Québec, le Ministère des Transports du Québec et la Fondation Communautaire Gaspésie-Les îles. Que toutes ces personnes physiques ou morales reçoivent l'expression de notre gratitude. L'auteur remercie tout particulièrement les deux évaluateurs du manuscrit, Markus Stoffel et Christian Bégin, ainsi que Louise Filion (rédactrice adjointe) et Sophie Roberge (coordonnatrice) pour les précieux commentaires et suggestions.

\section{RÉFÉRENCES}

Ancey, C., 1996. Guide neige et avalanches: connaissances, pratiques et sécurité. Edisud, Aix-en-Provence, $31 \mathrm{p}$.

Atlas du Bas-Saint-Laurent, 1998. Disponible en ligne à http ://atlasbsl. uqar.qc.ca/, page consultée le 3 mars 2008.

Bakkehoi, S., 1987. Snow avalanche prediction using a probabilistic method. In Proceedings of the Davos Symposium. International Association of the Hydrological Sciences, 162: 549-555.

Bigras, P. et Hébert, A., 1982. Vers un système météorologique de prévision des avalanches, mont Washington, New Hampshire. Université de Sherbrooke, $31 \mathrm{p}$.

Birkeland, K.W., 1998. Terminology and predominant processes associated with the formation of weak layers of near-surface faceted crystals in the mountain snowpack. Arctic and Alpine Research, 30: 193-199.

Birkeland, K.W., Mock, C.J. et Shinker, J.J., 2001. Avalanche extremes and atmospheric circulation patterns. Annals of Glaciology, 32: 135-140.

Boucher, D., 2000. Projet d'implantation d'un centre d'avalanche dans le parc de la Gaspésie. Municipalité Régionale de Comté Denis-Riverin, Sainte-Annedes-Monts, $26 \mathrm{p}$.

Boucher, D. et Gagnon, S., 2002. Modification de l'offre d'activités hivernales en vue d'une gestion sécuritaire du risque d'avalanche au mont Albert, parc de la Gaspésie. Centre d'avalanche de la Haute-Gaspésie, Sainte-Anne-desMonts, $10 \mathrm{p}$.

Boucher, D., Filion, L. et Hétu, B., 2003. Reconstitution dendrochronologique et fréquence des grosses avalanches de neige dans un couloir subalpin du mont Hog's Back, Gaspésie centrale (Québec). Géographie physique et Quaternaire, 57: 159-168.

Butler, D.R., 1986. Snow-avalanche hazards in Glacier National Park, Montana: meteorological and climatological aspects. Physical Geography, 7: 72-87.

Colbeck, S.C. et Jamieson, J.B., 2001. The formation of faceted layers above crusts. Cold Regions Science and Technology, 33: 247-252.

Conway, H., 2004. Storm Lewis: a rain-on-snow event on the Milford Road, New Zealand. In Proceedings of the International Snow Science Workshop 2004, Jackson Hole, p. 557-564.

Conway, H. et Raymond, C.F., 1993. Snow stability during rain. Journal of Glaciology, $30:$ 635-642. 
Conway, H. et Wilbour, C., 1999. Evolution of snow slope stability during storms. Cold Regions Science and Technology, 30:67-77.

Dubé, S., Filion, L. et Hétu, B., 2004. Tree-ring reconstruction of high-magnitude snow avalanches in the Northern Gaspé Peninsula, Québec. Arctic, Antarctic and Alpine Research, 36 : 541-550.

Environnement Canada, 2005a. Données climatiques en ligne. Disponible en ligne à http://www.climate.weatheroffice.ec.gc.ca/climateData/canada_f.html, page consultée le 3 mars 2008.

Environnement Canada, 2005b. Normales climatiques au Canada, période 1971-2000, stations de Mont-Louis, de Cap-Madeleine et de Cap-Chat. Disponible en ligne à http://www.climate.weatheroffice.ec.gc.ca/climate_ normals/index_f.html, page consultée le 3 mars 2008.

Environnement Canada, 2006. Atlas canadien d'énergie éolienne. Disponible en ligne à http://www.atlaseolien.ca/fr/index.php, page consultée le 3 mars 2008.

Fitzharris, B.B., 1981. Frequency and climatology of major avalanches at Roge Pass, 1909 to 1977. National Research Council of Canada, Ottawa, 42 p.

Gagnon, R.M., 1970. Climat des Chic-Chocs. Ministère des Richesses Naturelles, Québec, Rapport MP36, 103 p.

Gagnon, S., 2003. Avalanche incident comes as a Choc in Quebec. Avalanche News, $66: 52-53$

Gaumond, M. et Hamelin, L.-É., 1960. Note de périglaciaire comparé des monts Washington et Jacques-Cartier. Cahiers de Géographie de Québec, 7:217218.

Germain, D., 2005. Dynamique des avalanches de neige en Gaspésie, Québec, Canada. Thèse de doctorat, Université Laval, $173 \mathrm{p}$.

Germain, D., Filion, L. et Hétu, B., 2005. Snow avalanche activity after fire and logging disturbances, Northern Gaspé Peninsula, Québec, Canada. Canadian Journal of Earth Science, 42: 2103-2116.

Girard, J.-F. et Hétu, B., 1989. Le mont Albert, Parc de la Gaspésie: inventaire des avalanches et cartographie des zones à risque. Ministère du Loisir, de la Chasse et de la Pêche du Québec, Québec, 36 p.

Girard, J.-F. et Hétu, B., 1994. Les mouvements de masse en Gaspésie septentrionale. Hydro-Québec, Montréal, $45 \mathrm{p}$.

Groleau, M., 1994. Impacts géomorphologiques et écologiques des chutes de glace de paroi sur l'évolution d'un versant forestier, Mont-Saint-Pierre, Gaspésie septentrionale. Mémoire de maîtrise, Université Laval, 68 p.

Hägeli, P. et McClung, D.M., 2003. Avalanche characteristics of a transitional snow climate. Columbia Mountains, British Columbia. Cold Regions Science and Technology, 37: 255-276.

Henley, G., 1980. Quand ce n'est pas la mer, c'est la montagne. La Voix Gaspésienne, édition du 13 février, p. 4.

Hétu, B., 1986. L'influence du contexte géomorphologique quaternaire sur la dynamique postglaciaire des versants raides de la Gaspésie septentrionale. Thèse de doctorat, Université de Montréal, 568 p.

Hétu, B., 1990. Évolution récente d'un talus d'éboulis en milieu forestier, Gaspésie, Québec. Géographie physique et Quaternaire, 44 : 199-215.

Hétu, B., 1991. Éboulis stratifiés actifs près de Manche-d’Épée, Gaspésie (Québec, Canada). Zeitschrift für Geomorphologie, 35: 439-461.

Hétu, B., 1992. Coarse cliff-top aeolian sedimentation in Northern Gaspésie, Québec (Canada). Earth Surface Processes and Landforms, 17: 95-108.

Hétu, B., 1995. Le litage des éboulis stratifiés cryonivaux en Gaspésie (Québec, Canada): rôle de la sédimentation nivéo-éolienne et des transits supranivaux. Permafrost and Periglacial Processes, 6: 147-171.

Hétu, B. et Bergeron, A., 2004. Les avalanches au Québec: analyse des conditions météorologiques et des facteurs de terrain propices au déclenchement des avalanches. Programme conjoint de sécurité-avalanche au Québec, Secrétariat général de Recherche et Sauvetage Canada, 84 p.

Hétu, B. et Brown, K., 2006. Inventaire des avalanches mortelles au Québec depuis 1825. Avalanche.ca, 79: 56-59.

Hétu, B. et Gray, J.T., 1980. Évolution postglaciaire des versants de la région de Mont-Louis, Gaspésie, Québec. Géographie physique et Quaternaire, 34 187-208.
Hétu, B. et Vandelac, P., 1989. La dynamique des éboulis schisteux au cours de I'hiver, Gaspésie septentrionale, Québec. Géographie physique et Quaternaire, 43: 389-406

Hétu, B., Girard, J.-F. et Boisjoly, J. 1994. Les risques naturels reliés à la dynamique des versants dans le nord de la Gaspésie: zone littorale et mont Albert. Bulletin de l'Association québécoise pour l'étude du Quaternaire, 20: $9-15$

Höller, P., 1999. Weather and avalanches in Austria. The Avalanche Review, 17: 10 .

Jacob, N., 2001. Fréquence, intensité et déclenchement des coulées de débris en milieu forestier, Gaspésie septentrionale, Québec. Mémoire de maîtrise, Université Laval, 77 p.

Jamieson, B. et Geldsetzer, T., 1997. Avalanches au Canada. Volume 4: 19841996. Canadian Avalanche Association, Revelstoke, 210 p.

Jomelli, V., Delval, C., Grancher, D., Escande, S., Brunstein, D., Hétu, B., Filion, L. et Pech, P., 2007. Probabilistic analysis of recent snow avalanche activity and weather in the French Alps. Cold Region Science and Technology, 47: 180-192.

Larocque, S., Hétu, B. et Filion, L., 2001. Geomorphic and dendroecological impacts of slushflows in Central Gaspé Peninsula (Québec, Canada). Geografiska Annaler, 83A: 191-201.

Latulippe, L., 1971. De la neige presque partout au Québec. Le Soleil, édition du 21 décembre, p. 38

Lemieux, S., 2007. Cartographie et classification du terrain à potentiel avalancheux des Chic-Chocs, Québec, Canada, à l'aide d'un système d'information géographique. Mémoire de maîtrise, Université de Sherbrooke, 98 p.

Loup, J. et Lovie, C., 1966. Sur la fréquence des avalanches en haute Tarentaise. Revue de Géographie alpine, 55: 587-604.

McClung, D.M., 1999. The encounter probability for mountain slope hazards. Canadian Geotechnical Journal, 36: 1195-1196.

McClung, D.M. et Schaerer, P., 2006. The Avalanche Handbook. The Mountaineers Books, Seattle, $342 p$

Mock, C.J. et Birkeland, K.W., 2000. Snow avalanche climatology of the Western United States Mountain Ranges. Bulletin of the American Meteorological Society, $81: 2367-2392$.

Pelletier, M., 1997. Distribution des glaces de paroi en Gaspésie septentrionale dans le secteur compris entre Tourelle et vallée de Manche-d’Épée. Mémoire de baccalauréat, Université du Québec à Rimouski, 19 p.

Perla, R.I., 1970. On contributory factors in avalanche hazard evaluation. Canadian Geotechnical Journal, 7: 414-419.

Reardon, B.A., Fagre, D.B. et Steiner, R.W., 2004. Natural avalanches and transportation: a case study from Glacier National Park, Montana, U.S.A. In Proceedings of the International Snow Science Workshop 2004, Jackson Hole, p. 582-597.

Royer, A. et Lemieux, S., 2006. Mapping and classification of potential avalanche sites in the Chic-Chocs Mountains, Québec, Canada, using geographic information systems. In Proceedings of the International Snow Science Workshop 2006, Telluride, p. 91-98.

Talbot, M.-J., 2002. Caractérisation des couloirs d'avalanches dans le secteur du mont Logan, Est du Québec, et étude des facteurs contrôlant leur distribution spatiale. Mémoire de baccalauréat, Université du Québec à Rimouski, 76 p.

Transports Québec, 2005. Atlas des zones et sites d'avalanches de neige sur les routes 132 et 198 en Haute-Gaspésie. Ministère des Transports du Québec, Québec, 90 p.

Veillette, J. et Cloutier, M., 1993. Géologie des formations en surface, Gaspésie, Québec. Commission géologique du Canada, Ottawa, Carte 1804A, échelle $1 / 250000$

Ward, R.G.W., 1984. Avalanche prediction in Scotland: II. Development of a predictive model. Applied Geography, 4 : 109-133. 